\title{
First-principles method for electron-phonon coupling and electron mobility Applications to two-dimensional materials
}

\author{
Gunst, Tue; Markussen, Troels; Stokbro, Kurt; Brandbyge, Mads
}

Published in:

Physical Review B

Link to article, DOI:

10.1103/physrevb.93.035414

Publication date:

2016

Document Version

Publisher's PDF, also known as Version of record

Link back to DTU Orbit

Citation (APA):

Gunst, T., Markussen, T., Stokbro, K., \& Brandbyge, M. (2016). First-principles method for electron-phonon coupling and electron mobility: Applications to two-dimensional materials. Physical Review B, 93(3), [035414]. https://doi.org/10.1103/physrevb.93.035414

\section{General rights}

Copyright and moral rights for the publications made accessible in the public portal are retained by the authors and/or other copyright owners and it is a condition of accessing publications that users recognise and abide by the legal requirements associated with these rights.

- Users may download and print one copy of any publication from the public portal for the purpose of private study or research.

- You may not further distribute the material or use it for any profit-making activity or commercial gain

- You may freely distribute the URL identifying the publication in the public portal 


\title{
First-principles method for electron-phonon coupling and electron mobility: Applications to two-dimensional materials
}

\author{
Tue Gunst,,${ }^{1, *}$ Troels Markussen, ${ }^{2}$ Kurt Stokbro, ${ }^{2}$ and Mads Brandbyge ${ }^{1}$ \\ ${ }^{1}$ Department of Micro- and Nanotechnology (DTU Nanotech), Center for Nanostructured Graphene (CNG), \\ Technical University of Denmark, DK-2800 Kgs. Lyngby, Denmark \\ ${ }^{2}$ QuantumWise A/S, Fruebjergvej 3, Postbox 4, DK-2100 Copenhagen, Denmark \\ (Received 6 November 2015; revised manuscript received 17 December 2015; published 8 January 2016)
}

\begin{abstract}
We present density functional theory calculations of the phonon-limited mobility in $n$-type monolayer graphene, silicene, and $\mathrm{MoS}_{2}$. The material properties, including the electron-phonon interaction, are calculated from first principles. We provide a detailed description of the normalized full-band relaxation time approximation for the linearized Boltzmann transport equation (BTE) that includes inelastic scattering processes. The bulk electron-phonon coupling is evaluated by a supercell method. The method employed is fully numerical and does therefore not require a semianalytic treatment of part of the problem and, importantly, it keeps the anisotropy information stored in the coupling as well as the band structure. In addition, we perform calculations of the low-field mobility and its dependence on carrier density and temperature to obtain a better understanding of transport in graphene, silicene, and monolayer $\mathrm{MoS}_{2}$. Unlike graphene, the carriers in silicene show strong interaction with the out-of-plane modes. We find that graphene has more than an order of magnitude higher mobility compared to silicene in the limit where the silicene out-of-plane interaction is reduced to zero (by substrate interaction, clamping, or similar). If the out-of-plane interaction is not actively reduced, the mobility of silicene will essentially be zero. For $\mathrm{MoS}_{2}$, we obtain several orders of magnitude lower mobilities compared to graphene in agreement with other recent theoretical results. The simulations illustrate the predictive capabilities of the newly implemented BTE solver applied in simulation tools based on first-principles and localized basis sets.
\end{abstract}

DOI: 10.1103/PhysRevB.93.035414

\section{INTRODUCTION}

Two-dimensional (2D) materials are promising candidates for future electronic devices [1-6]. Examples of three such materials are illustrated in Fig. 1, showing a monolayer of graphene [Fig. 1(a)], its silicon-based counterpart silicene [7-10] [Fig. 1(b)], and monolayer $\mathrm{MoS}_{2}$ [Fig. 1(c)]. In such systems, two dimensionality allows very precise control of the carrier density by a gate which enables tuning of the electron-phonon interaction [3]. Electron-phonon interaction in graphene has been studied previously [11-22], but with the recent advances in fabrication of devices based on other $2 \mathrm{D}$ materials, like the first demonstration of a silicene transistor [4], further studies of interaction phenomena in 2D materials are necessary.

When comparing the electrical performance of devices one often considers the carrier mobility of the materials. Mobility is a key parameter for the semiconductor industry describing the motion of electrons when an electric field is applied. Experiments can approach the "intrinsic" phonon-limited mobility by several means. One experiment combines defect-free edge contacting [23] of gate-tunable graphene electrodes with $\mathrm{MoS}_{2}$ encapsulated in hexagonal boron nitride layers [24]. Several other experiments screen the scattering from charged impurities by a high- $\kappa$ gate dielectrics [25] or through device suspension in high- $\kappa$ liquids $[15,26,27]$. In general, van der Waals heterostructures may pave the way for devices with reduced extrinsic scattering, such as charged impurity scattering, rendering modeling

\footnotetext{
*Tue.Gunst@nanotech.dtu.dk
}

of electron-phonon scattering in these devices even more important.

Conventional mobility modeling usually considers effective mass approximations [28] in the case of semiconductors or linear bands for semimetals [11-13] combined with empirical deformation potentials and semianalytical solutions of the Boltzmann equation [13,29]. The Boltzmann theory is a useful approach to model the low-field/linear-response mobility [29-31], as well as the high-field transport through Monte Carlo simulations $[14,32,33]$. Several studies have examined effects related to screening [34,35], scattering from out-ofplane vibrations [36], and performed atomistic calculations of the mobility from tight-binding [37-39] as well as electronphonon interaction's role in facilitating interlayer conduction [40] and current-induced heating [41,42]. Density functional theory (DFT) and atomistic methods can be used to assess the electronic structure and electron-phonon coupling in novel 2D materials where fitted deformation potential parameters are not available [43-49].

Recently, several groups have combined DFT with density functional perturbation theory $[43,44,50-52]$ to evaluate the mobility from first principles. Parameter-free methods can be used to address how close experiments are to ideal conductivities and if further optimization of fabrication techniques and device designs for novel 2D materials could improve device performance. In addition, first-principles calculations of the bulk electron-phonon interaction may be used for comparing deformation potential values to those obtained from experiments $[53,54]$ and used to conclude which scattering effects are dominant. The dominant effect is not always directly clear from experiments, and published deformation potentials can vary significantly [13]. 
(a)

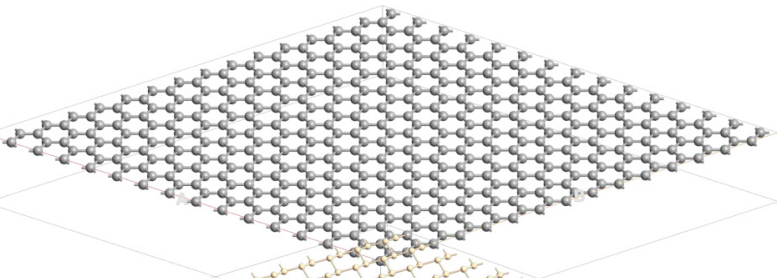

(b)

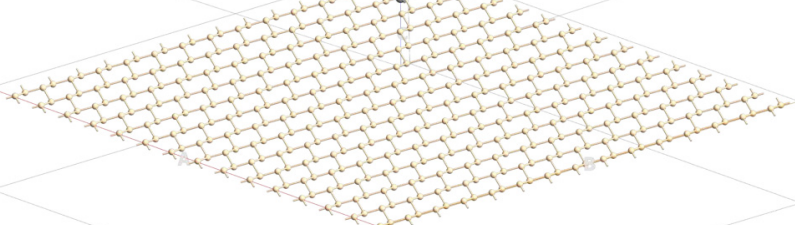

(c)

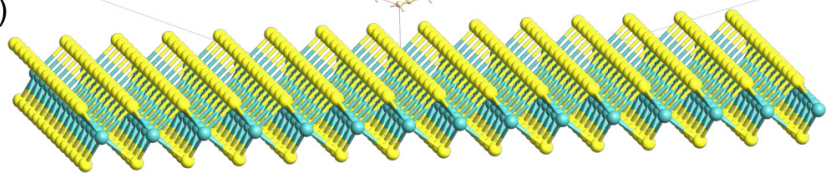

FIG. 1. The monolayered two-dimensional systems that are considered are (a) graphene, (b) silicene, and (c) $\mathrm{MoS}_{2}$.

Several techniques which differ from contacted electrical measurements are in development that can provide detailed knowledge of the electron-phonon interaction; broadening of Raman peaks [55], kinks in the angle-resolved photoemission spectra [56], and nondestructive optical methods [57]. Hereby, a first-principles method to evaluate bulk electron-phonon interactions may provide useful data for comparison with experiments.

Methods to obtain the bulk electron-phonon interactions include Wannier functions together with a generalized Fourier interpolation scheme [58], perturbation theory and empirical pseudopotentials [59], finite differences in the projectoraugmented wave method [60], and density functional perturbation theory $[50,51,61,62]$.

We will later give a simple derivation of an expression for the bulk electron-phonon coupling applicable in any setup based on localized basis sets. From this we have implemented a supercell method used to calculate the bulk electron-phonon interaction employing finite differences. This method is analogous to that used for calculating the phonon dispersion both in terms of methodology and computational cost.

In this paper, we present a detailed description of the implementation of the bulk electron-phonon coupling, and a Boltzmann transport equation (BTE) solver in the Atomistix ToolKit (ATK) simulation tool [63] We apply atomistic simulations with ATK to study electron-phonon coupling in 2D materials from first principles. We formulate a normalized full-band relaxation time approximation (RTA) for the linearized BTE that includes inelastic scattering processes. The bulk electronphonon methodology employed makes simulations possible that do not require a semianalytic treatment of part of the problem and, importantly, we keep the anisotropy information stored in the coupling as well as the band structure. In addition, we perform calculations of the low-field mobility and its dependence on carrier density and temperature to obtain a better understanding of transport in graphene, silicene, and $\mathrm{MoS}_{2}$.

Despite the fact that several papers have presented schemes for obtaining electron-phonon coupling and mobilities from first-principles calculations, only few applications exist and the methods are by no means standard. The focus of our work is to make an efficient and practical scheme for such calculations in order to make them as accessible as performing a band-structure calculation. In the Methods section, we will discuss the differences in our technical implementation compared to previous work, and here we only briefly mention some general advantages of our work which we believe will be important for the widespread usage. The method is based on localized basis sets which allow for exploiting locality in real space and we have made a great effort of optimizing the implementation and apply efficient parallelization and interpolation schemes. The method is therefore fast and able to handle large systems. Second, it is highly accurate and as we will show in this paper, it gives results consistent with previous DFT simulations. Finally, it is implemented in a versatile framework with an easy to use python interface which allows for performing all the different parts of the calculations with a single script, thus, it is easy to set up the calculation and it requires minimal human interference for performing the calculation.

The paper is organized as follows. In Sec. II, we present the theoretical and numerical methods used. We derive expressions for the linearized BTE and the bulk electron-phonon interaction implemented in the ATK simulation tool. In Sec. III, we present our results for the bulk electron-phonon coupling in graphene, silicene, and $\mathrm{MoS}_{2}$. In addition, we discuss the dependence of the mobility on the carrier density and temperature for all three materials. Finally, the results are summarized and discussed in Sec. IV.

\section{METHODS}

In the diffusive transport limit, the mobility can be obtained by solving the semiclassical BTE for the electronic distribution function $f\left(\varepsilon_{\mathbf{k} n}\right)=f_{\mathbf{k} n}$ :

$$
\frac{\partial f_{\mathbf{k} n}}{\partial t}+\mathbf{v}_{\mathbf{k} n} \cdot \nabla_{\mathbf{r}} f_{\mathbf{k} n}+\frac{\mathbf{F}}{\hbar} \cdot \nabla_{\mathbf{k}} f_{\mathbf{k} n}=\left.\frac{\partial f_{\mathbf{k} n}}{\partial t}\right|_{\text {coll }} .
$$

Here, $\mathbf{k}, n$ labels the $k$ point and band index, respectively. The velocity is defined as $\mathbf{v}_{\mathbf{k} n}=1 / \hbar \nabla_{\mathbf{k}} \varepsilon_{\mathbf{k} n}$ and $\mathbf{F}=q(\mathbf{E}+\mathbf{v} \times \mathbf{B})$ gives the external force. The right-hand side in Eq. (1) describes different sources of scattering and dissipation that drives the system towards steady state. In the case of a homogeneous system, zero magnetic field, and a time-independent electric field in the steady-state limit, the BTE simplifies to

$$
\frac{q \mathbf{E}}{\hbar} \cdot \nabla_{\mathbf{k}} f_{\mathbf{k} n}=\left.\frac{\partial f_{\mathbf{k} n}}{\partial t}\right|_{\text {coll }} .
$$

Assuming instantaneous, single collisions, which are independent of the driving force, the collision integral can be expressed using transition rates $P_{\mathbf{k k}^{\prime}}^{n n^{\prime}}$ :

$$
\begin{aligned}
\left.\frac{\partial f_{\mathbf{k} n}}{\partial t}\right|_{\text {coll }}=- & \sum_{\mathbf{k}^{\prime} n^{\prime}}\left[f_{\mathbf{k} n}\left(1-f_{\mathbf{k}^{\prime} n^{\prime}}\right) P_{\mathbf{k} \mathbf{k}^{\prime}}^{n n^{\prime}}\right. \\
& \left.-f_{\mathbf{k}^{\prime} n^{\prime}}\left(1-f_{\mathbf{k} n}\right) P_{\mathbf{k}^{\prime} \mathbf{k}}^{n^{\prime} n}\right] .
\end{aligned}
$$

The transition rate due to phonon scattering from a state $|\mathbf{k} n\rangle$ to $\left|\mathbf{k}^{\prime} n^{\prime}\right\rangle$ is obtained from Fermi's golden rule 
(FGR):

$$
\begin{aligned}
P_{\mathbf{k} \mathbf{k}^{\prime}}^{n n^{\prime}}= & \frac{2 \pi}{\hbar} \sum_{\mathbf{q}, \lambda}\left|g_{\mathbf{k} \mathbf{q}}^{\lambda n n^{\prime}}\right|^{2}\left[n_{\mathbf{q}}^{\lambda} \delta\left(\epsilon_{\mathbf{k}^{\prime} n^{\prime}}-\epsilon_{\mathbf{k} n}-\hbar \omega_{\mathbf{q} \lambda}\right) \delta_{\mathbf{k}^{\prime}, \mathbf{k}+\mathbf{q}}\right. \\
& \left.+\left(n_{-\mathbf{q}}^{\lambda}+1\right) \delta\left(\epsilon_{\mathbf{k}^{\prime} n^{\prime}}-\epsilon_{\mathbf{k} n}+\hbar \omega_{-\mathbf{q} \lambda}\right) \delta_{\mathbf{k}^{\prime}, \mathbf{k}-\mathbf{q}}\right]
\end{aligned}
$$

describing absorption (first term) and emission (last term) of a phonon. The last term includes spontaneous emission which remains at zero temperature. The sum runs over phonon momentum (q) and phonon branch index ( $\lambda$ ). In Eq. (4), we have explicitly stated the momentum conservation coming from the bulk electron-phonon interaction matrix element. We have implemented a supercell method to calculate the bulk electron-phonon interaction $g_{\mathbf{k q}}^{\lambda n n^{\prime}}$, employing finite differences in a localized basis set (derived in Sec. II C).

We will assume unperturbed phonons and apply the equilibrium Bose-Einstein distributions $n_{\mathbf{q}}^{\lambda}=n_{\mathbf{q}}^{0, \lambda}$, in which case $n_{-\mathbf{q}}^{0, \lambda}=n_{\mathbf{q}}^{0, \lambda}$ since $\omega_{-\mathbf{q} \lambda}=\omega_{\mathbf{q} \lambda}$. The transition rates $P_{\mathbf{k k}^{\prime}}^{\lambda n n^{\prime}}$ and $P_{\mathbf{k}^{\prime} \mathbf{k}}^{\lambda n^{\prime} n}$ are linked through the "detailed balance equation" [30]

$$
\left[f_{\mathbf{k} n}^{0}\left(1-f_{\mathbf{k}^{\prime} n^{\prime}}^{0}\right) P_{\mathbf{k k}^{\prime}}^{n n^{\prime}}-f_{\mathbf{k}^{\prime} n^{\prime}}^{0}\left(1-f_{\mathbf{k} n}^{0}\right) P_{\mathbf{k}^{\prime} \mathbf{k}}^{n^{\prime} n}\right]=0 .
$$

Here, $f^{0}$ is the equilibrium Fermi distribution function. This equation secures that $\left.\frac{\partial f_{\mathbf{k} n}}{\partial t}\right|_{\text {coll }}=0$ in equilibrium.

We linearize the BTE in the electric field. The left-hand side of the BTE, Eq. (2), is approximated to linear order in the electric field by changing to the equilibrium distribution:

$$
\frac{q \mathbf{E}}{\hbar} \cdot \nabla_{\mathbf{k}} f_{\mathbf{k} n} \approx \frac{q \mathbf{E}}{\hbar} \cdot \nabla_{\mathbf{k}} f_{\mathbf{k} n}^{0}=q \mathbf{E} \cdot \mathbf{v}_{\mathbf{k} n} \frac{\partial f_{\mathbf{k} n}^{0}}{\partial \epsilon_{\mathbf{k} n}} .
$$

The right-hand side is linearized by assuming a form of the distribution function that is linear in the electric field. Defining a generalized transport relaxation time $[30,44] \tau_{\mathbf{k} n}$, so that

$$
f_{\mathbf{k} n}=f_{\mathbf{k} n}^{0}+q \mathbf{E} \cdot \mathbf{v}_{\mathbf{k} n} \tau_{\mathbf{k} n}\left(-\frac{\partial f_{\mathbf{k} n}^{0}}{\partial \epsilon_{\mathbf{k} n}}\right),
$$

and combining and inserting Eqs. (3), (4), (5), (6) in Eq. (2), we arrive at the linearized BTE

$$
\begin{aligned}
1= & \sum_{\mathbf{k}^{\prime} n^{\prime}} P_{\mathbf{k} \mathbf{k}^{\prime}}^{n n^{\prime}} \frac{\left(1-f_{\mathbf{k}^{\prime} n^{\prime}}^{0}\right)}{\left(1-f_{\mathbf{k}^{n} n}^{0}\right)} \\
& \times\left[\tau_{\mathbf{k} n}-\tau_{\mathbf{k}^{\prime} n^{\prime}} \frac{n_{\mathbf{k}^{\prime} n^{\prime}}}{n_{\mathbf{k} n}} \frac{f_{\mathbf{k} n}^{0}\left(1-f_{\mathbf{k} n}^{0}\right)}{f_{\mathbf{k}^{\prime} n^{\prime}}^{0}\left(1-f_{\mathbf{k}^{\prime} n^{\prime}}^{0}\right)}\right],
\end{aligned}
$$

where we defined the direction projections $n_{\mathbf{k} n}=\hat{\mathbf{E}} \cdot \hat{\mathbf{v}}_{\mathbf{k} n}$. Equation (8) is still a full integral equation. However, several approximations, termed relaxation time approximations (RTA), exist throughout literature $[43,64,65]$ to reduce the problem to a $\mathbf{k}^{\prime}$-space integration. For instance, the term in the brackets in Eq. (8) is replaced by $\tau_{\mathbf{k} n}$ times the nonnormalized factors $\left[1-\frac{\mathbf{k} \cdot \mathbf{k}^{\prime}}{k^{2}}\right],\left[1-\frac{\mathbf{v}_{\mathbf{k}^{\prime} n^{\prime} \cdot \mathbf{v} \mathbf{k}^{\prime}}}{\left|\mathbf{v}_{\mathbf{k} n}\right|^{2}}\right]$ or the normalized factors [1- $\left.\frac{\mathbf{k} \cdot \mathbf{k}^{\prime}}{k k^{\prime}}\right]$ and infrequently [1- $\frac{\mathbf{V}_{\mathbf{k}^{\prime} n^{\prime}} \cdot \mathbf{v}_{\mathbf{k} n}}{\left.\left|\mathbf{V}_{\mathbf{k}^{\prime} n}\right| \mathbf{v}_{\mathbf{k} n}\right]}$ [65]. The non-normalized conditions are related to the assumption that $\tau_{\mathbf{k}^{\prime} n^{\prime}} \approx \tau_{\mathbf{k} n}$ while the normalized expressions are related to the assumption that $\tau_{\mathbf{k}^{\prime} n^{\prime}}\left|\mathbf{v}_{\mathbf{k}^{\prime} n^{\prime}}\right| \approx \tau_{\mathbf{k} n}\left|\mathbf{v}_{\mathbf{k} n}\right|$, and in both cases that the last Fermi factor is equal to unity. The expressions based on group velocities have the advantage that they do not depend on the chosen reference $\mathbf{k}$ point. In addition, non-normalized expressions may lead to unphysical negative momentum relaxation times. Here, we define a normalized full-band RTA of the linearized BTE, including inelastic scattering processes, as

$$
\frac{1}{\tau_{\mathbf{k} n}}=\sum_{\mathbf{k}^{\prime} n^{\prime}} \frac{\left(1-f_{\mathbf{k}^{\prime} n^{\prime}}^{0}\right)}{\left(1-f_{\mathbf{k} n}^{0}\right)}\left[1-\cos \left(\theta_{\mathbf{k k}^{\prime}}\right)\right] \mathrm{P}_{\mathbf{k} \mathbf{k}^{\prime}}^{\mathrm{nn}} .
$$

Here, the scattering angle is defined by [66]

$$
\cos \left(\theta_{\mathbf{k k}^{\prime}}\right)=\frac{n_{\mathbf{k}^{\prime} n^{\prime}}}{n_{\mathbf{k} n}}=\frac{\mathbf{v}_{\mathbf{k}^{\prime} n^{\prime}} \cdot \mathbf{v}_{\mathbf{k} n}}{\left|\mathbf{v}_{\mathbf{k}^{\prime} n^{\prime}}\right|\left|\mathbf{v}_{\mathbf{k} n}\right|} .
$$

For an angle-independent transition rate then small-angle scattering, where $\cos \left(\theta_{\mathbf{k k}^{\prime}}\right) \approx 1$, does not obstruct the flow of electrons, whereas large-angle scattering, where $\cos \left(\theta_{\mathbf{k k}^{\prime}}\right) \approx$ -1 , significantly increases resistivity. However, the selection rules stored in the bulk electron-phonon coupling matrix element complicate this general trend. The current density is related to the average velocity $\mathbf{J}=q n_{0}\left\langle\mathbf{v}_{\mathbf{k} n}\right\rangle$, obtained from the nonequilibrium distribution function in Eq. (7). From the transport relaxation time [Eq. (9)], we then evaluate the low-field electron mobility [64]

$$
\mu_{e}=-2 q \frac{\sum_{\mathbf{k} n \in c}\left|\mathbf{v}_{\mathbf{k} n}\right|^{2} \frac{\partial f_{\mathbf{k} n}^{0}}{\partial \epsilon_{\mathbf{k} n}} \tau_{\mathbf{k} n}}{n_{0}},
$$

where a factor of 2 accounts for the spin degeneracy. The hole mobility is obtained by replacing the electron carrier density $n_{0}=\sum_{\mathbf{k} n \in c} f_{\mathbf{k} n}^{0}$, and the summation over conduction bands (c) with the hole carrier density $p_{0}=\sum_{\mathbf{k} n \in v}\left(1-f_{\mathbf{k} n}^{0}\right)$, and a summation over valance bands $(v)$. The total conductivity is given by $\sigma=q n_{0} \mu_{e}+q p_{0} \mu_{h}$.

We also mention the difference of the transport scattering time discussed here versus the lifetime of electronic quasiparticles. The lifetime can be measured by angle-resolved photoemission spectroscopy and is evaluated as

$$
\begin{aligned}
\frac{1}{\tau_{\mathbf{k} n}^{l}}= & \frac{2 \pi}{\hbar} \sum_{\mathbf{k}^{\prime} \mathbf{q} n^{\prime} \lambda}\left|g_{\mathbf{k} \mathbf{q}}^{\lambda n n^{\prime}}\right|^{2} \\
& \times\left[\left(n_{\mathbf{q}}^{\lambda}+f_{\mathbf{k}^{\prime} n^{\prime}}^{0}\right) \delta\left(\epsilon_{\mathbf{k}^{\prime} n^{\prime}}-\epsilon_{\mathbf{k} n}-\hbar \omega_{\mathbf{q} \lambda}\right) \delta_{\mathbf{k}^{\prime}, \mathbf{k}+\mathbf{q}}\right. \\
& \left.+\left(1+n_{\mathbf{q}}^{\lambda}-f_{\mathbf{k}^{\prime} n^{\prime}}^{0}\right) \delta\left(\epsilon_{\mathbf{k}^{\prime} n^{\prime}}-\epsilon_{\mathbf{k} n}+\hbar \omega_{\mathbf{q} \lambda}\right) \delta_{\mathbf{k}^{\prime}, \mathbf{k}-\mathbf{q}}\right] .
\end{aligned}
$$

Performing the $\mathbf{k}^{\prime}$ sum, we obtain

$$
\begin{aligned}
\frac{1}{\tau_{\mathbf{k} n}^{l}}= & \frac{2 \pi}{\hbar} \sum_{\mathbf{q} n^{\prime} \lambda}\left|g_{\mathbf{k q}}^{\lambda n n^{\prime}}\right|^{2}\left[\left(n_{\mathbf{q}}^{\lambda}+f_{\mathbf{k}+\mathbf{q} n^{\prime}}^{0}\right) \delta\left(\epsilon_{\mathbf{k}+\mathbf{q} n^{\prime}}-\epsilon_{\mathbf{k} n}-\hbar \omega_{\mathbf{q} \lambda}\right)\right. \\
& \left.+\left(1+n_{\mathbf{q}}^{\lambda}-f_{\mathbf{k}-\mathbf{q} n^{\prime}}^{0}\right) \delta\left(\epsilon_{\mathbf{k}-\mathbf{q} n^{\prime}}-\epsilon_{\mathbf{k} n}+\hbar \omega_{\mathbf{q} \lambda}\right)\right] .
\end{aligned}
$$

This equation can be derived by keeping only all terms proportional to $f_{\mathbf{k} n}$ in Eq. (3) and corresponds approximately to neglecting the scattering angle transport factor $[1-\cos (\theta)]$ in Eq. (9) $[17,64,67]$.

\section{A. Numerical details}

The full $\mathbf{k}$-dependent scattering rate, in contrast to a simplified energy-dependent expression, leads to several numerical complications which have not been addressed in the literature to the best of our knowledge. Therefore, we will outline the main technicalities needed to account for the anisotropy information stored in the full band structure and the electronphonon coupling. We represent the delta functions in Eq. (4) 
by Lorentzians $\delta(\epsilon) \approx \frac{1}{\pi} \frac{\gamma / 2}{(\gamma / 2)^{2}+\epsilon^{2}}$, with a finite broadening $\gamma$. Consequently, we find that it is important to evaluate the Fermi factors $f_{\mathbf{k}^{\prime} n^{\prime}}^{0}$ at the exact final energy, $\epsilon_{\mathbf{k} n} \pm \hbar \omega$ as opposed to $\epsilon_{\mathbf{k} \pm \mathbf{q} n}$, to get correct results. This also means that absorption and emission terms need to be handled independently when it comes to the peculiar Fermi prefactor in Eq. (9). In addition, we rewrite the prefactor in the case of absorption, to avoid numerical instabilities:

$$
\frac{\left(1-f_{\mathbf{k}^{\prime} n^{\prime}}^{0}\right)}{\left(1-f_{\mathbf{k} n}^{0}\right)}=\frac{f_{\mathbf{k} n}^{0}}{f_{\mathbf{k}^{\prime} n^{\prime}}^{0}} \frac{1+n_{B}\left(\epsilon_{\mathbf{k}^{\prime} n^{\prime}}-\epsilon_{\mathbf{k} n}\right)}{n_{B}\left(\epsilon_{\mathbf{k}^{\prime} n^{\prime}}-\epsilon_{\mathbf{k} n}\right)} .
$$

This secures a stable denominator since absorption will dominate the low-energy spectrum $\epsilon<\mu_{F}$ where $f^{0} \rightarrow 1$ and $1-f^{0} \rightarrow 0$. Considering the $\mathbf{k}$ and $\mathbf{q}$ grids, a conversion factor of $\Delta \mathbf{q} / \Delta \mathbf{k}$ is needed if the $\mathbf{k}$ grid and $\mathbf{q}$ grid are not equivalent. One can show that if the two grids are equivalent, the full linearized BTE [Eq. (8)] simplifies to a linear matrix equation. However, it is advantageous to allow for different grid resolutions in order to apply smart choosing of the grids and resulting simulation speedup. A fine resolution of the final-state $\mathbf{q}$ grid secures a correct result for each $\mathbf{k}$ point even at a rough $\mathbf{k}$ grid. Our approach has therefore been to use fine $\mathbf{q}$ grids with the possibility of interpolation to even higher resolution for all $\mathbf{q}$-dependent variables.

As a final remark, we mention that the velocities $\mathbf{v}_{\mathbf{k} n}, \mathbf{v}_{\mathbf{k}^{\prime} n^{\prime}}$ are obtained from perturbation theory. From a change $d \mathbf{k}_{\alpha}$, we obtain the derivative

$$
\frac{d \mathbf{H}_{\mathbf{k}}}{d \mathbf{k}_{\alpha}}=\sum_{I} i \mathbf{R}_{I, \alpha} \mathbf{H}_{v \mu} e^{i \mathbf{k} \cdot \mathbf{R}_{I}}
$$

of the Hamiltonian matrix in a basis of localized orbitals $(\nu, \mu)$, and correspondingly for the overlap matrix $\mathbf{S}$ by replacing $\mathbf{H} \rightarrow \mathbf{S}$. Here, $I$ labels the unit cells, with lattice vectors $\mathbf{R}_{I}$, of a supercell discussed thoroughly in the next sections. A perturbation calculation then gives

$$
\mathbf{v}_{\mathbf{k} n, \alpha}=\frac{1}{\hbar} \frac{d \epsilon_{\mathbf{k} n}}{d \mathbf{k}_{\alpha}}=\frac{1}{\hbar}\left\langle n \mathbf{k}\left|\frac{d \mathbf{H}_{\mathbf{k}}}{d \mathbf{k}_{\alpha}}-\epsilon_{\mathbf{k} n} \frac{d \mathbf{S}_{\mathbf{k}}}{d \mathbf{k}_{\alpha}}\right| n \mathbf{k}\right\rangle,
$$

where $\epsilon_{\mathbf{k} n}$ and $|n \mathbf{k}\rangle$ are the energy and Bloch state of band $n$ at wave vector $\mathbf{k}$. We hereby avoid finite-difference errors from crossing bands when the bands are not sorted correctly.

\section{B. Phonons}

The phonon polarization vectors $\left(\mathbf{e}_{\mathbf{q}}^{\lambda}\right)$ and energies $\hbar \omega_{\mathbf{q}, \lambda}$ are obtained as solutions to the equation

$$
\mathbf{D}(\mathbf{q}) \mathbf{e}_{\mathbf{q}}^{\lambda}=\omega_{\mathbf{q}, \lambda}^{2} \mathbf{e}_{\mathbf{q}}^{\lambda},
$$

where $\mathbf{q}$ is the phonon momentum and $\lambda$ labels the phonon branch (band) index and $\mathbf{D}(\mathbf{q})$ is the Fourier transformed dynamical matrix. We initially compute the real-space dynamical matrix from a standard finite-difference approach [68], where the elements are given by

$$
\begin{aligned}
D_{i \mu j \nu} & =\frac{1}{\sqrt{M_{i} M_{j}}} \frac{\partial^{2} E_{\mathrm{tot}}(\mathbf{R})}{\partial \mathbf{x}_{i \nu} \partial \mathbf{x}_{j \mu}} \\
& \approx \frac{1}{\sqrt{M_{i} M_{j}}} \frac{F_{j \mu}\left(+\Delta \mathbf{x}_{i \nu}\right)-F_{j \mu}\left(-\Delta \mathbf{x}_{i \nu}\right)}{2 \Delta \mathbf{x}_{i \nu}},
\end{aligned}
$$

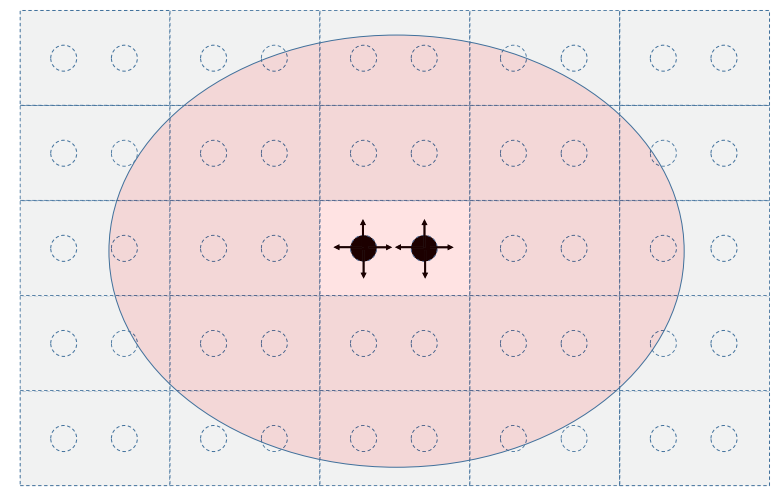

FIG. 2. Schematic illustration of the supercell method. A unit cell is repeated $5 \times 5$ times. Only the atoms in the central unit cell are being displaced, while forces are evaluated on all the atoms in the supercell. Outside a certain range (ellipsoidal area), the forces are zero.

where $i, j$ are atom indices and $\mu, v$ denote Cartesian directions. $E_{\text {tot }}(\mathbf{R})$ is the total energy written as a function of all atomic coordinates. A change of variable from first-order derivative of energy to force was applied in Eq. (17), where the force $F_{j \mu}\left(+\Delta \mathbf{x}_{i v}\right)$ is acting on atom $j$ in direction $\mu$ when atom $i$ is displaced by $\Delta \mathbf{x}_{i v}$ in direction $\nu$. The approximate equality sign indicates the approximation inherent in the first-order finite-difference method.

When computing the forces, we first construct a supercell by repeating the unit cell $\left(n_{A}, n_{B}, n_{C}\right)$ times along the directions of the primitive lattice vectors. Second, we perform the finitedifference derivative by calculating the forces in the entire supercell, while only displacing the atom in the central unit cell, as schematically shown in Fig. 2.

The normalized phonon eigenmodes in Eq. (16) are dimensionless. The transformation to modes with physical dimension is $\mathbf{u}_{\mathbf{q}}^{\lambda}=l_{q} \mathbf{e}_{\mathbf{q}}^{\lambda}$, where the characteristic length is calculated from the polarization vectors and the diagonal mass matrix $\mathbf{m}$ :

$$
l_{\mathbf{q}}^{\lambda}=\sqrt{\frac{\hbar}{2 \omega_{\lambda} \mathbf{e}_{\mathbf{q}}^{\lambda \dagger} \cdot \mathbf{m} \cdot \mathbf{e}_{\mathbf{q}}^{\lambda}} .}
$$

\section{Bulk electron-phonon coupling}

We here provide a simple derivation of the bulk electronphonon coupling in the case of a localized basis by applying the periodicity of the problem. An equivalent expression was previously obtained in the Wannier basis method in Ref. [58]. We want to calculate the coupling matrix element between Bloch states $|n \mathbf{k}\rangle$ and $\left|n^{\prime} \mathbf{k}^{\prime}\right\rangle$ due to a phonon with momentum $\mathbf{q}$ and branch index $\lambda$ perturbing the Hamiltonian:

$$
g_{\mathbf{k k}^{\prime} \mathbf{q}}^{\lambda n n^{\prime}}=\left\langle n^{\prime} \mathbf{k}^{\prime}\left|\delta \hat{H}_{\mathbf{q} \lambda}\right| n \mathbf{k}\right\rangle .
$$

The perturbation to the Hamiltonian can be expressed as

$$
\delta \hat{H}_{\mathbf{q} \lambda}=l_{\mathbf{q}}^{\lambda} \sum_{\alpha} \sum_{I} \frac{\partial \hat{H}}{\partial \mathbf{x}_{I, \alpha}} \mathbf{e}_{\mathbf{q}, I, \alpha}^{\lambda},
$$

where the $I$ sum runs over the periodic unit cells and the $\alpha$ sum runs over the spatial degrees of freedom (atom index and Cartesian direction) within each cell. Using the Bloch 
periodicity of the phonon polarization vector, we can rewrite this as

$$
\delta \hat{H}_{\mathbf{q} \lambda}=l_{\mathbf{q}}^{\lambda} \sum_{\alpha} \mathbf{e}_{\mathbf{q}, \alpha}^{\lambda} \sum_{I} \frac{\partial \hat{H}}{\partial \mathbf{x}_{I, \alpha}} e^{i \mathbf{q} \cdot \mathbf{R}_{I}}
$$

where now $\mathbf{e}_{\mathbf{q}, \alpha}^{\lambda}$ are components of the polarization vector in the unit cell with index " 0 " (the reference cell). The unit cell with index $I$ is displaced from the reference cell by lattice vector $\mathbf{R}_{I}$.

We evaluate the derivative of the Hamiltonian in a similar manner as the dynamical matrix described above. A unit cell is repeated to form a supercell, but only the atoms in the central unit cell are displaced. The terms that contribute to the derivative are the (local) effective potential $V_{\text {local }}(\mathbf{r})$ and the nonlocal (NL) Kleinmann-Bylander term $V_{\mathrm{NL}}$. Details of the derivative of the Hamiltonian are given in Appendix.

The electronic Bloch states are expressed as

$$
|n \mathbf{k}\rangle=\frac{1}{\sqrt{N}} \sum_{I} \sum_{\mu} c_{n, \mathbf{k}}^{\mu} e^{i \mathbf{k} \cdot \mathbf{r}}\left|\phi_{\mu} ; \mathbf{R}_{I}\right\rangle,
$$

where the $I$ sum runs over $N$ unit cells in the macroscopic system, $\mu$ labels the basis orbitals within a single unit cell, $c_{n, \mathbf{k}}^{\mu}$ are expansion coefficients, and $\left|\phi_{\mu} ; \mathbf{R}_{I}\right\rangle$ is the $\mu$ th basis orbital in the unit cell displaced from the reference cell by the lattice vector $\mathbf{R}_{I}$. Inserting (21) and (22) in (19) we get

$$
\begin{aligned}
g_{\mathbf{k k}^{\prime} \mathbf{q}}^{\lambda n n^{\prime}}= & \frac{l_{\mathbf{q}}^{\lambda}}{N} \sum_{I J K} \sum_{\mu \nu} \sum_{\alpha} e^{-i \mathbf{k}^{\prime} \cdot \mathbf{R}_{J}} e^{i \mathbf{k} \cdot \mathbf{R}_{K}}\left(c_{n^{\prime}, \mathbf{k}^{\prime}}^{\nu}\right)^{*} c_{n, \mathbf{k}}^{\mu} \\
& \times \mathbf{e}_{\mathbf{q}, \alpha}^{\lambda}\left\langle\phi_{\nu} ; \mathbf{R}_{J}\left|\frac{\partial \hat{H}}{\partial \mathbf{x}_{I, \alpha}} e^{i \mathbf{q} \cdot \mathbf{R}_{I}}\right| \phi_{\mu} ; \mathbf{R}_{K}\right\rangle .
\end{aligned}
$$

Due to the periodicity of the system, the derivative of the Hamiltonian matrix with respect to atom positions in $\mathbf{R}_{I}$ can be shifted as follows:

$$
\left\langle\phi_{\nu} ; \mathbf{R}_{I}+\mathbf{R}_{m}\left|\frac{\partial \hat{H}}{\partial \mathbf{x}_{I, \alpha}}\right| \phi_{\mu} ; \mathbf{R}_{I}+\mathbf{R}_{l}\right\rangle=\left\langle\phi_{\nu} ; \mathbf{R}_{m}\left|\frac{\partial \hat{H}}{\partial \mathbf{x}_{0, \alpha}}\right| \phi_{\mu} ; \mathbf{R}_{l}\right\rangle,
$$

where we defined the relative vectors $\mathbf{R}_{m / l}$ connecting cell $K, J$ to the cell $I$. As for the force derivative calculation described in Sec. II B, the derivative of the Hamiltonian will also be nonzero in a region around the atoms being displaced. The $J, K$ sums in Eq. (23) which run over all cells in the macroscopic sample can be limited to the cells included in the supercell calculation of the $\partial \hat{H} / \partial \mathbf{x}_{0, \alpha}$. We thus replace the $J, K$ sums with sums over neighboring cells $m, l$ relative to $I$ in Eq. (23):

$$
\begin{aligned}
g_{\mathbf{k k}^{\prime} \mathbf{q}}^{\lambda n n^{\prime}}= & \frac{l_{\mathbf{q}}^{\lambda}}{N} \sum_{I m l} \sum_{\mu \nu} \sum_{\alpha} e^{-i \mathbf{k}^{\prime} \cdot\left(\mathbf{R}_{I}+\mathbf{R}_{m}\right)} e^{i \mathbf{k} \cdot\left(\mathbf{R}_{I}+\mathbf{R}_{l}\right)} e^{i \mathbf{q} \cdot \mathbf{R}_{I}} \\
& \times\left(c_{n^{\prime}, \mathbf{k}^{\prime}}^{\nu}\right)^{*} c_{n, \mathbf{k}}^{\mu} \mathbf{e}_{\mathbf{q}, \alpha}^{\lambda}\left\langle\phi_{\nu} ; \mathbf{R}_{m}\left|\frac{\partial \hat{H}}{\partial \mathbf{x}_{0, \alpha}}\right| \phi_{\mu} ; \mathbf{R}_{l}\right\rangle,
\end{aligned}
$$

where the derivative of the Hamiltonian is only carried out for spatial degrees of freedom $(\alpha)$ in the reference unit cell. The $I$ sum can now be carried out to simply give a factor of $\sum_{I} e^{i\left(\mathbf{k}-\mathbf{k}^{\prime}+\mathbf{q}\right) \cdot \mathbf{R}_{I}}=N \delta_{\mathbf{k}^{\prime}, \mathbf{k}+\mathbf{q}}$ which enforces momentum conservation. Defining $g_{\mathbf{k} \mathbf{k}^{\prime} \mathbf{q}}^{\lambda n n^{\prime}}=g_{\mathbf{k q}}^{\lambda n n^{\prime}} \delta_{\mathbf{k}^{\prime}, \mathbf{k}+\mathbf{q}}$, Eq. (24) is then simplified to the final expression for the bulk electron-phonon coupling in a supercell setup:

$$
\begin{aligned}
g_{\mathbf{k q}}^{\lambda n n^{\prime}}= & l_{\mathbf{q}}^{\lambda} \sum_{m l} \sum_{\mu \nu} \sum_{\alpha} e^{i \mathbf{k} \cdot \mathbf{R}_{l}-i(\mathbf{k}+\mathbf{q}) \cdot \mathbf{R}_{m}}\left(c_{n^{\prime}, \mathbf{k}+\mathbf{q}}^{\nu}\right)^{*} c_{n, \mathbf{k}}^{\mu} \\
& \times \mathbf{e}_{\mathbf{q}, \alpha}^{\lambda}\left\langle\phi_{\nu} ; \mathbf{R}_{m}\left|\frac{\partial \hat{H}}{\partial \mathbf{x}_{0, \alpha}}\right| \phi_{\mu} ; \mathbf{R}_{l}\right\rangle .
\end{aligned}
$$

In summary, Eq. (25) provides a procedure for calculating the bulk electron-phonon coupling in any localized basis setup: One has to evaluate the finite differences of a supercell Hamiltonian where atoms in the center cell are displaced and a summation over unit cells is performed with corresponding phase factors [69].

\section{SIMULATIONS AND RESULTS}

The simulations were performed using the ATK DFT code with the PBE-GGA functional for exchange correlation in the cases of graphene and silicene, and LDA in the case of $\mathrm{MoS}_{2}$. In all cases, we use a double-zeta-polarized (DZP) basis set. The real-space grid cutoff was $110 \mathrm{Ha}$. The geometries were relaxed until all forces were smaller than $0.001 \mathrm{eV} / \AA$, and $51 \times 51$ in-plane $k$ points were used in the electronic-structure calculations. A vacuum gap of $30 \AA$ was used in the direction normal to the material plane and Dirichlet boundary conditions was used in the Poisson equation for this direction. The bulk electron-phonon interaction and phonon dispersion was obtained from a $11 \times 11$ supercell calculation in the case of graphene and a $9 \times 9$ supercell for silicene and $\mathrm{MoS}_{2}$. The delta functions in Eq. (4) were numerically represented by Lorentzians with a broadening of $\gamma=3 \mathrm{meV}$.

\section{A. Band structures}

One obtains linear valence and conduction bands near the Dirac point $K$ in both graphene and silicene, as shown by the band structures in Fig. 3(a). We obtain Fermi velocities of $0.9 \times 10^{6} \mathrm{~m} / \mathrm{s}$ and $0.57 \times 10^{6} \mathrm{~m} / \mathrm{s}$ of graphene and silicene, respectively. Both materials have six phonon branches. The three acoustic modes will dominate the low-temperature scattering where two modes (LA, TA) have a linear $q$ dependence and the third out-of-plane acoustic (ZA) mode has a $\mathrm{q}^{2}$ dependence near the Brillouin zone center [70] [see Fig. 3(b)]. We obtain sound velocities of $20.4(12.6) \times 10^{3} \mathrm{~m} / \mathrm{s}$ for the LA (TA) mode of graphene and $9.1(6.1) \times 10^{3} \mathrm{~m} / \mathrm{s}$ for the LA (TA) mode of silicene. $\mathrm{MoS}_{2}$ is found to be a direct-gap semiconductor [71,72] with a band gap of $1.89 \mathrm{eV}$ [see Fig. 3(c)]. The electron and phonon band structures, in Figs. 3(c) and 3(d), are consistent with previous theoretical results [60]. $\mathrm{MoS}_{2}$ has three acoustic and six optical branches. The three acoustic branches are the in-plane longitudinal acoustic (LA), the transverse acoustic (TA), as well as the out-of-plane acoustic (ZA) modes. We obtain sound velocities of $6.6(4.2) \times 10^{3} \mathrm{~m} / \mathrm{s}$ for the LA (TA) mode of $\mathrm{MoS}_{2}$. The six optical branches are two in-plane longitudinal optical ( $\mathrm{LO}_{1}$, $\left.\mathrm{LO}_{2}\right)$, two in-plane transverse optical $\left(\mathrm{TO}_{1}, \mathrm{TO}_{2}\right)$, and two out-of-plane optical $\left(\mathrm{ZO}_{1}, \mathrm{ZO}_{2}\right)$ modes. The two lowest optical branches $\left(\mathrm{LO}_{1}, \mathrm{TO}_{1}\right)$ are nonpolar modes which do not couple to the charge carriers. The next two branches $\left(\mathrm{LO}_{2}, \mathrm{TO}_{2}\right)$ are polar optical modes where the Mo and $\mathrm{S}$ atoms vibrate in 
(a)

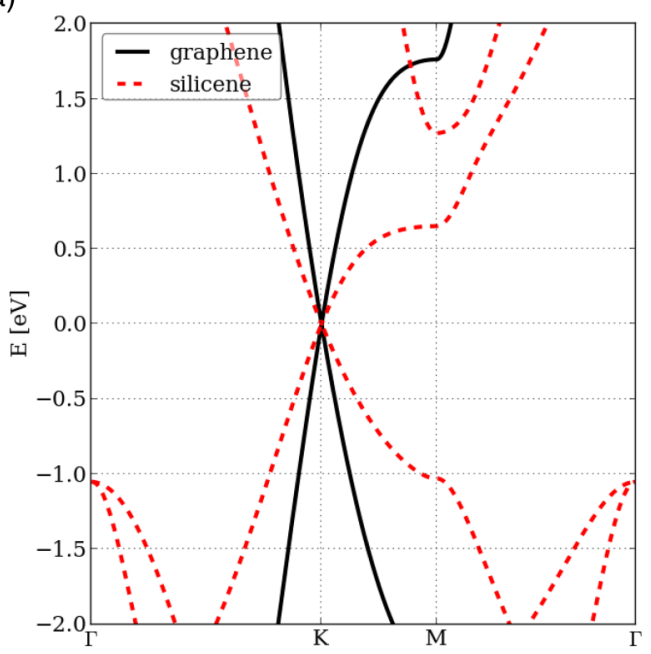

(c)

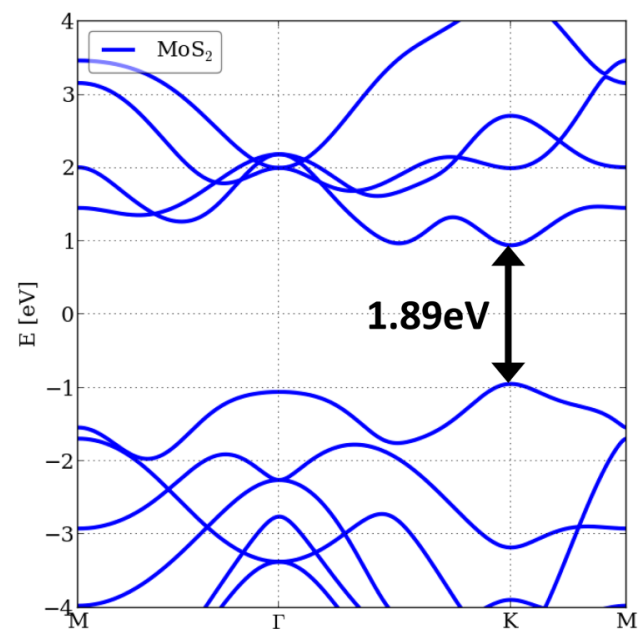

(b)

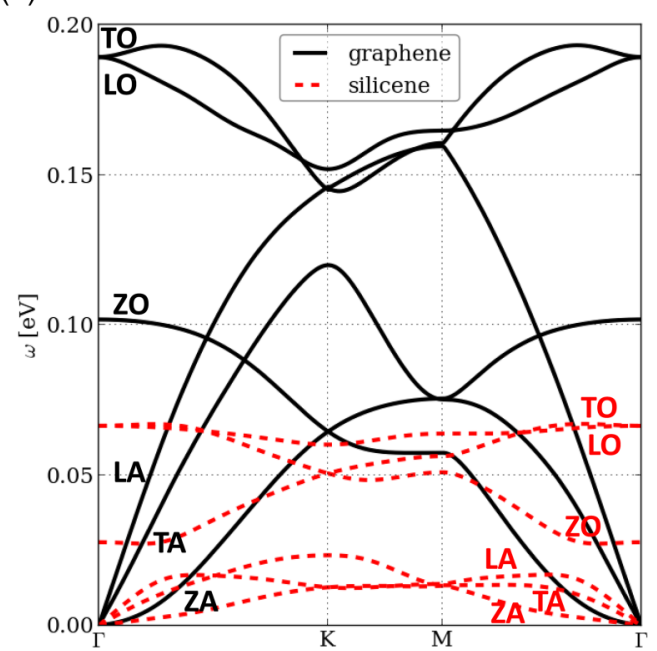

(d)

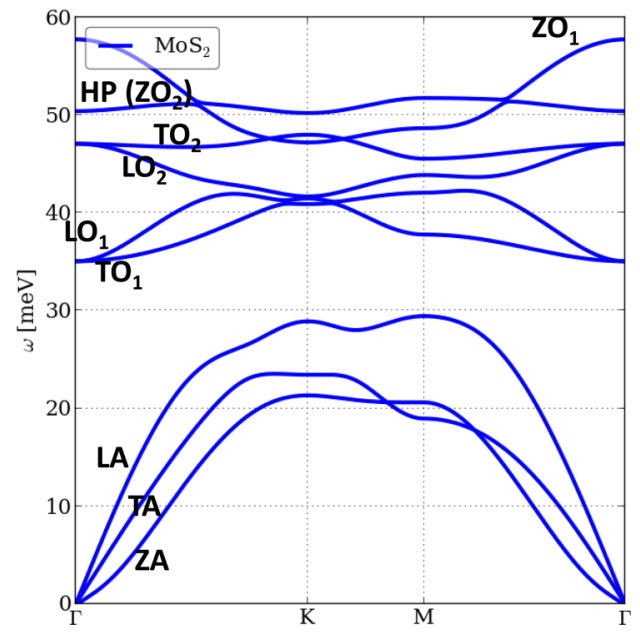

FIG. 3. Electron band structure of (a) graphene and silicene and (c) $\mathrm{MoS}_{2}$ and phonon dispersion of (b) graphene and silicene and (d) $M o S_{2}$.

counterphase. The dispersionless out-of-plane mode $\mathrm{ZO}_{2}$ is also called the homopolar mode. It is characteristic of layered structures and is related to fluctuations in the layer thickness [73]. For further discussion of phonon modes in $\mathrm{MoS}_{2}$, we refer the reader to Refs. [74,75].

\section{B. Bulk electron-phonon coupling}

We now turn to the calculated bulk electron-phonon coupling. It is common practice to plot the coupling matrix element $g_{\mathbf{k q}}^{\lambda n n^{\prime}}$ in Eq. (25), for a fixed $\mathbf{k}$ point as a function of $\mathbf{q}$. In this way, one can visualize the detailed suppression of the scattering, as opposed to a constant or linear-in- $q$ deformation potential, depending on the symmetry of the involved phonon and electron states. The units are converted to $\mathrm{eV} / \AA$, often used for extracted deformation potentials from experiments, by dividing by the characteristic length prefactor $l_{\mathbf{q}}^{\lambda}$ in Eq. (25).

In Fig. 4, we illustrate the $\mathbf{q}$ variation of the bulk electronphonon interaction obtained for the four modes coupling with electrons in graphene. The atomic motion of the considered mode is illustrated as an insert in the upper right corner, by arrows indicating the atomic displacement. The interaction is obtained around $300 \mathrm{meV}$ from the Dirac $K$ point. Comparing the interaction with two previously published results $[17,43]$, we find that it is slightly higher for the acoustic modes (between 10\%-20\%) and very close in magnitude for the optical modes (within 10\%), but in both cases with the same symmetry. The maximal values for the TO and LO modes are in energy units approximately $0.4-0.5 \mathrm{eV}$ for comparison. Importantly, we see that the coupling elements are highly anisotropic. In the case of acoustic modes, we see that backscattering $\left(q_{x}<0, q_{y} \approx 0\right)$ is suppressed for the LA mode, while the situation is reversed for the TA mode where forward scattering is suppressed. In addition, other directions with complete suppression also appear and the $q$ dependence is highly nontrivial. In general, the anisotropy and scattering suppression of the bulk electron-phonon coupling is determined by the combined symmetry of both phonon and electronic states.

Unlike graphene, the carriers in silicene display a strong interaction with the ZA mode [47] (see Fig. 5). This is related to the buckling of the silicene sheet, where one basis atom is displaced approximately $0.44 \AA$ out of plane, hence breaking the planar symmetry. Otherwise, we see that 

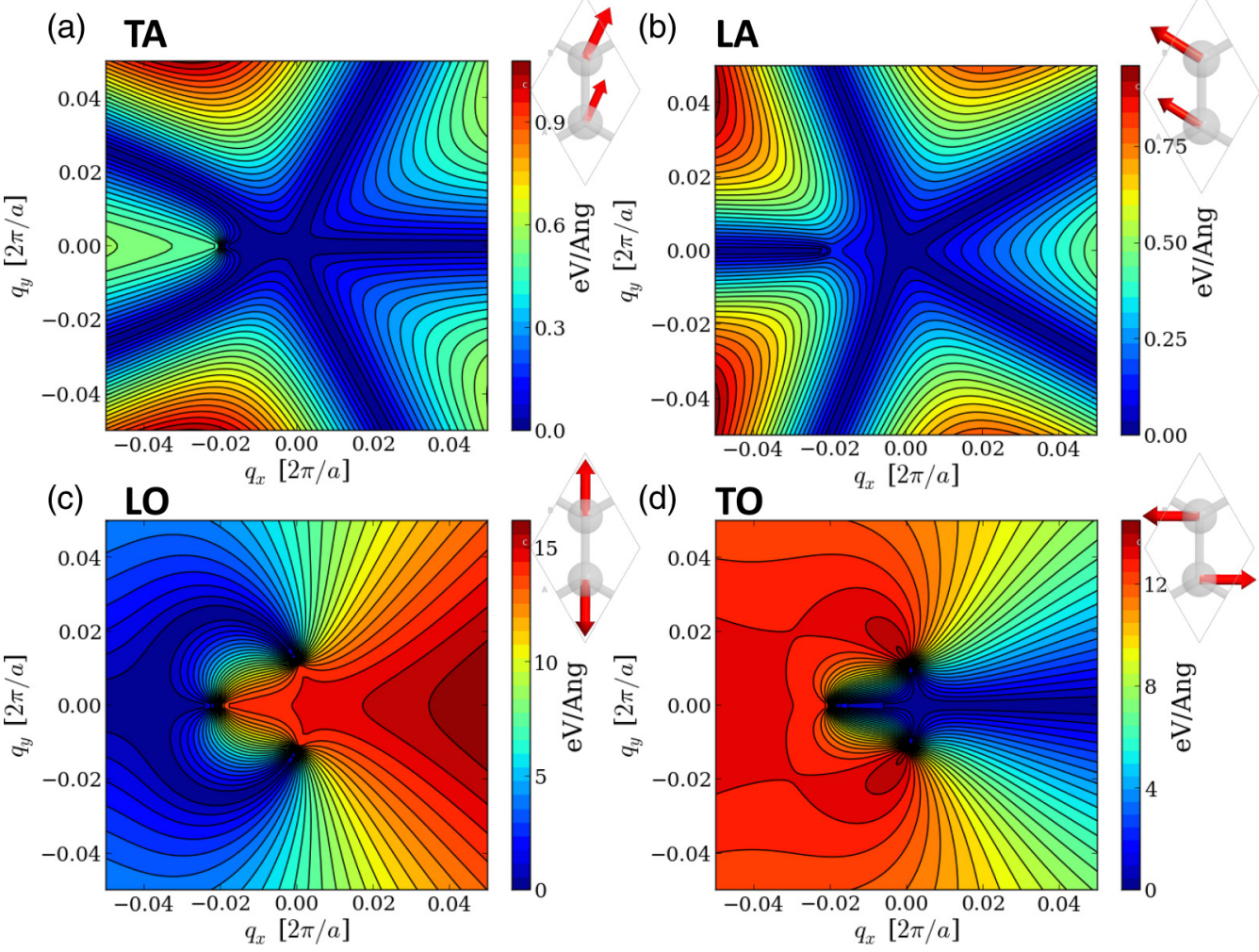

FIG. 4. Bulk electron-phonon coupling in graphene for the four modes with nonzero coupling. The interaction is illustrated as a function of phonon $\mathbf{q}$ vector at a $\mathbf{k}$ point shifted $300 \mathrm{meV}$ from the Dirac $K$ point towards the $\Gamma$ point. We refer the reader to Ref. [17] for a detailed discussion of the interpretation of the plots for the TA and LA modes. The scattering rate is obtained as integrals around the constant energy circles on the Dirac cone satisfying $\varepsilon_{\mathbf{k}^{\prime}}=\varepsilon_{\mathbf{k}} \pm \hbar \omega$ [cf. Eqs. (4) and (9)]. Insets: phonon modes are visualized by arrows indicating the atomic displacements.

the situation for the acoustic modes is similar to that of graphene. Backscattering is suppressed for the LA mode, while the situation is reversed for the TA mode where forward scattering is suppressed. Again, other directions with complete suppression of the scattering also appear.

It is important to realize that the reduction of the symmetry of the lattice leads to additional scattering mechanisms and modes interacting with the charge carriers. This was the case for silicene where there was no planar symmetry, and it is even more so for the case of $\mathrm{MoS}_{2}$ which lacks inversion symmetry. In the last mentioned case, all kinds of electronphonon coupling may take place, such as different orders of deformation potential, piezoelectric and Fröhlich couplings. This makes a strong case for a fully numerical solution of the BTE together with a first-principles method to evaluate the bulk electron-phonon interactions. We avoid having a high number of free parameters in an analytical model and instead every type of interaction is directly taken care of. In Fig. 6, we show the bulk electron-phonon interaction obtained for the five modes coupling with electrons in $\mathrm{MoS}_{2}$. Unlike graphene and silicene, which are semimetals, $\mathrm{MoS}_{2}$ is a semiconductor and the interaction is evaluated at the conduction band minimum since only $n$ doping is relevant in $\mathrm{MoS}_{2}$. Again, we find very anisotropic couplings where the symmetry compares well to previously published results [60].

The coupling with the TA, LA, and TO modes is of the same order of magnitude as in Ref. [60] [cf. Figs. 6(a)-6(c)], but we obtain a somewhat lower coupling for the homopolar mode (approximately 65\% lower) [see Fig. 6(d)]. Figure 6(e) shows the Fröhlich interaction for the polar optical $\mathrm{LO}_{2}$ mode. The Fröhlich interaction is difficult to converge with respect to supercell size. In the long-wavelength limit, this element should increase linearly $[60,73]$. We find that the peaks increase in magnitude by approximately $4 \%$ and move toward $|\mathbf{q}| \rightarrow 0$ as expected if the supercell size is increased from $9 \times 9$ to $15 \times 15$. Therefore, this interaction is only partially accounted for, except for the low-temperature limit where the optical $\mathrm{LO}_{2}$ mode is not occupied. Several recent papers propose methods for evaluating the long-range couplings from DFT by using Born effective charges [76,77], which relies on a partitioning into long- and short-ranged interactions. It should be mentioned that long-ranged couplings related to macroscopic $E$ fields has to be screened separately in a similar partitioning scheme to account for the additional screening imposed by the surrounding dielectric encapsulation used for 2D materials to screen charged impurities.

\section{Scattering rates}

We obtain the scattering rate by integrating the linearized BTE within the RTA [cf. Eq. (9)]. The electron-phonon coupling is evaluated for every $\mathbf{k}$ point up to an energy cutoff in a $100 \times 100 \mathbf{q}$ mesh. The coupling, energies, and velocities were subsequently interpolated to twice this q-space 
(a) TA
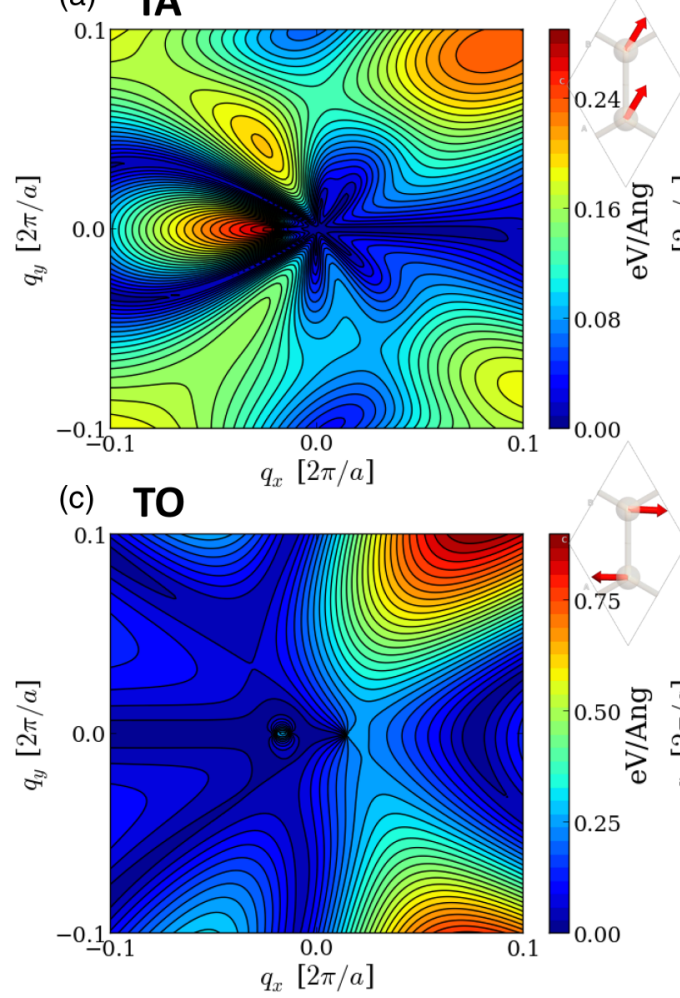

(b) LA

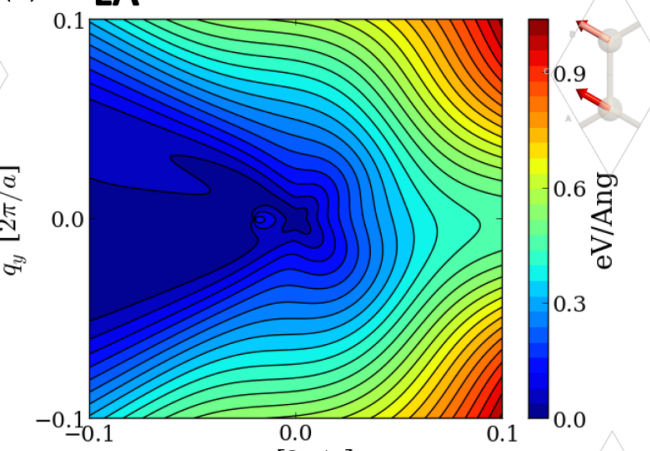

(d) $q_{x}[2 \pi / a]$

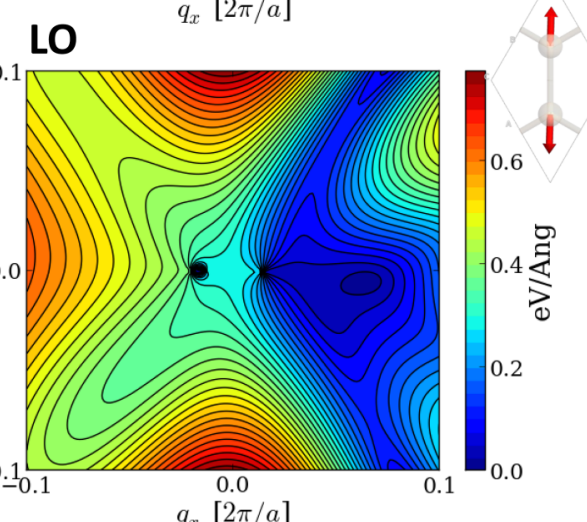

(e) ZA
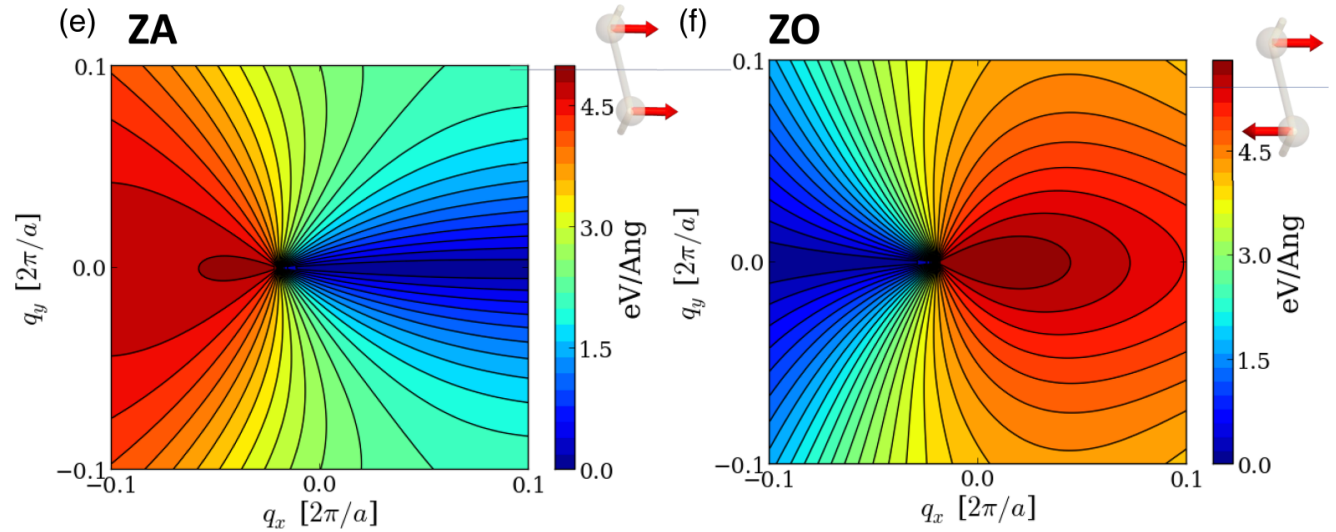

FIG. 5. Bulk electron-phonon coupling in silicene. Unlike graphene also the out-of-plane modes (ZA, ZO) couple significantly with electrons. The interaction is illustrated as a function of phonon $\mathbf{q}$ vector at a $\mathbf{k}$ point shifted $110 \mathrm{meV}$ from the Dirac $K$ point towards the $\Gamma$ point. The scattering rate is obtained as integrals around the constant energy circles on the Dirac cone satisfying $\varepsilon_{\mathbf{k}^{\prime}}=\varepsilon_{\mathbf{k}} \pm \hbar \omega$ [cf. Eqs. (4) and (9)]. Insets: phonon modes are visualized by arrows indicating the atomic displacements.

resolution before the BTE was solved. In Fig. 7, we show the result obtained for graphene. Below the Bloch-Grüneisen temperature (approximate $57 \sqrt{n} \mathrm{~K}$ for graphene with the carrier density measured in units of $10^{12} \mathrm{~cm}^{-2}$ ), only those phonons with short $\mathbf{q}$ are effectively excited. This manifest itself in the dip in the scattering rate around the Fermi level. We clearly see the expected low-temperature Bloch-Grüneisen dips around the Fermi level (100 $\mathrm{meV}$ in the present case) and the opening of optical phonon interaction (emission) at $\mu_{F}+\hbar \omega$ [Fig. 7(a)]. It is illustrative to plot the rate along a single $\mathbf{k}$ line as in Fig. 7(a). However, the full two-dimensional dependency is needed to capture the anisotropy of the scattering rate. Figure 7(b) illustrates the scattering rate and inverse lifetime of the LA and TA modes found in a full two-dimensional $\mathbf{k}$ mesh. The spread of the points illustrates a significant dependence on directions of the scattering rate. This is further highlighted in Figs. 7(c) and 7(d) where the scattering rate was interpolated to clearly illustrate the anisotropy of especially the LA and TA modes. Part of the anisotropy originates from the bulk electron-phonon coupling. This is the main contribution to the anisotropy of the inverse lifetime. However, the anisotropy is further amplified by the transport scattering angle $1-\cos (\theta)$ in Eq. (9), which is seen by comparing scattering rate and inverse lifetime in Fig. 7(b).

We conclude that the scattering rate depends significantly on the k-space directions. In addition, our implementation gives results for the graphene scattering rate that are consistent with previous theoretical results in the low-temperature Bloch-Grüneisen regime, as well as the high-temperature 


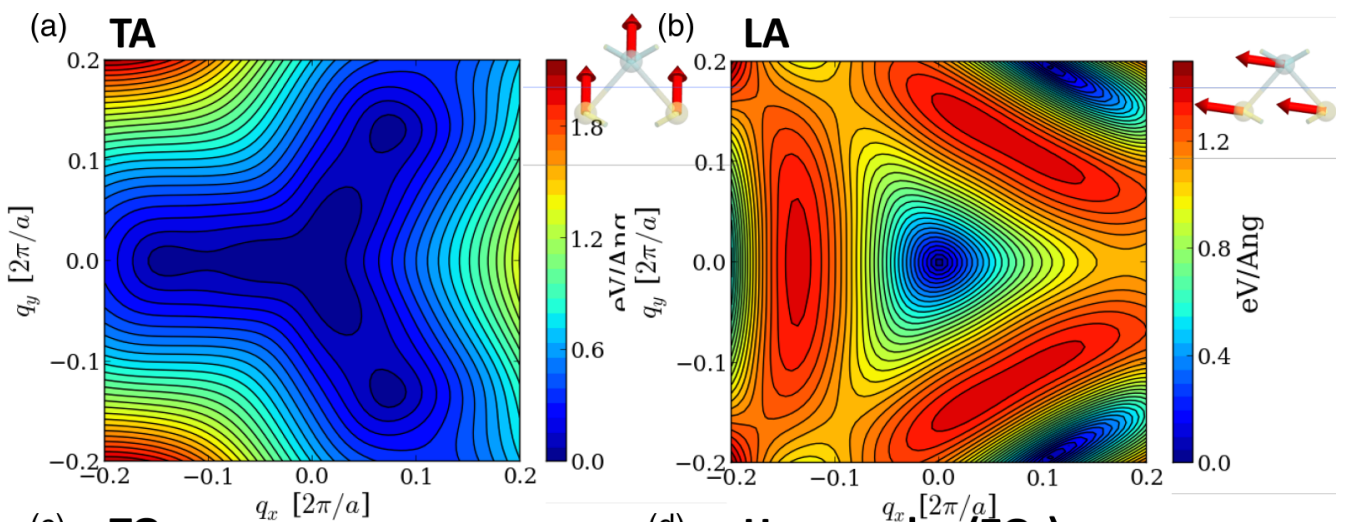

(c) $\mathrm{TO}_{2}$

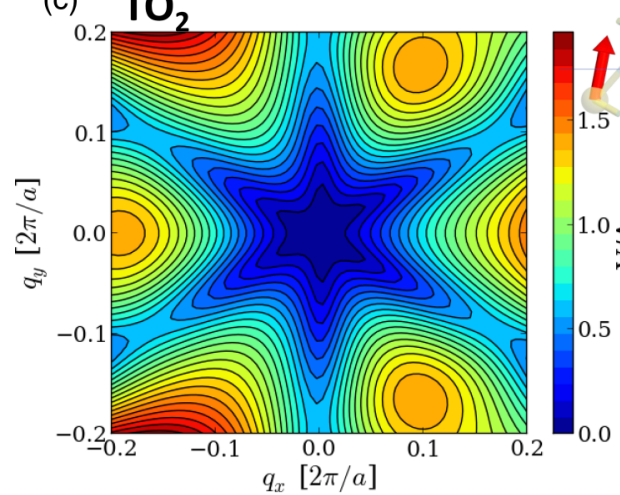

(e) (d)

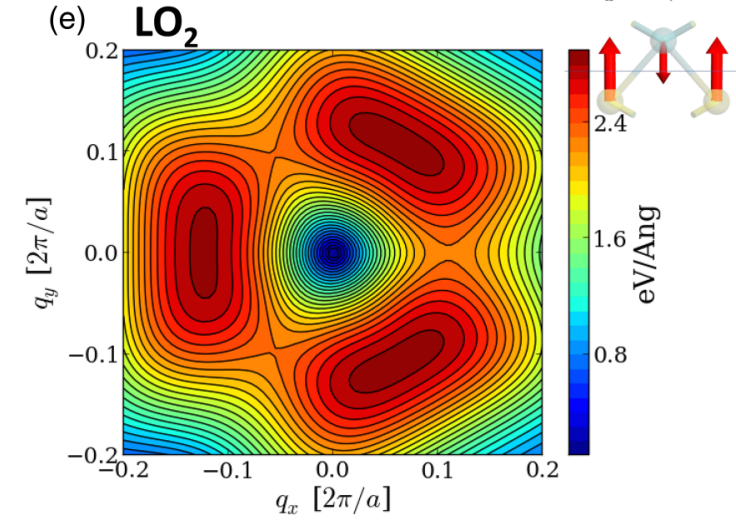

FIG. 6. Bulk electron-phonon coupling in $\mathrm{MoS}_{2}$. The interaction is illustrated as a function of phonon $\mathbf{q}$ vector at the conduction band minimum $K$ point. Insets: phonon modes are visualized by arrows indicating the atomic displacements.

equipartition regime where the scattering rate should depend linearly on energy $[13,60]$.

Previous studies have calculated the lifetime of carriers in silicene [47]. In Fig. 8, we show the scattering rate along a single $\mathbf{k}$ line for silicene and $\mathrm{MoS}_{2}$. We find a significant scattering with the ZA mode for silicene. Surprisingly, the scattering rate with the ZA mode is two to three orders of magnitude larger than the coupling with the remaining modes. This was not captured in the previous study in Ref. [47] due to a linear dispersion model applied for the quadratic ZA mode. However, this mode is difficult to describe accurately. The electron-phonon interaction is only partly screened in the present formalism and the quadratic dispersion of the ZA modes results in a constant density of states which is therefore not able to cut off long-wavelength interactions. One could hope that the scattering rate with the out-of-plane ZA mode would be significantly reduced due to interaction with a substrate and a more precise description of screening. The origin of the strong ZA coupling was very recently shown by Fischetti et al. [78] to be quite general for materials with broken planar symmetry and lies in the divergence of the thermal population of the flexural ZA phonons. In that paper, they also discuss how screening and reasonable long-wavelength cutoffs introduced by disorder will not be sufficient to reduce the coupling with the ZA mode. However, it is still interesting to compare the obtained value with and without the ZA coupling to illustrate the maximal gain by clamping of the sample. The obtained mobilities should in any case be taken as upper limits.

For both silicene and $\mathrm{MoS}_{2}$, we find that the rates only change slightly if the Fermi level is reduced further. For $\mathrm{MoS}_{2}$ the scattering rate is almost independent of $\mu_{F}$ for $\mu_{F}<E_{c}$; only the scattering rates for the $\mathrm{LO}_{2}$ and LA modes reduce slightly at high energy. Unlike the scattering related 
(a)

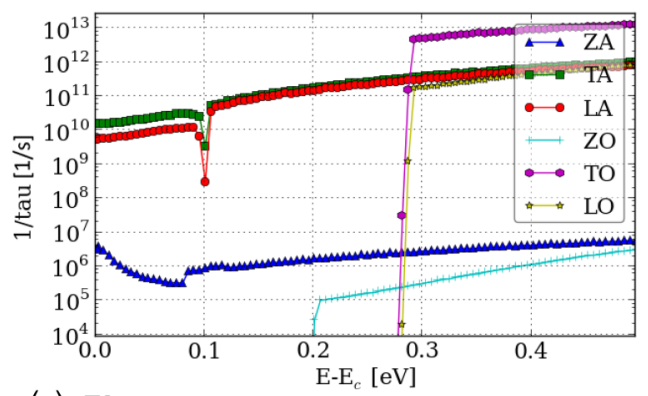

(c) TA

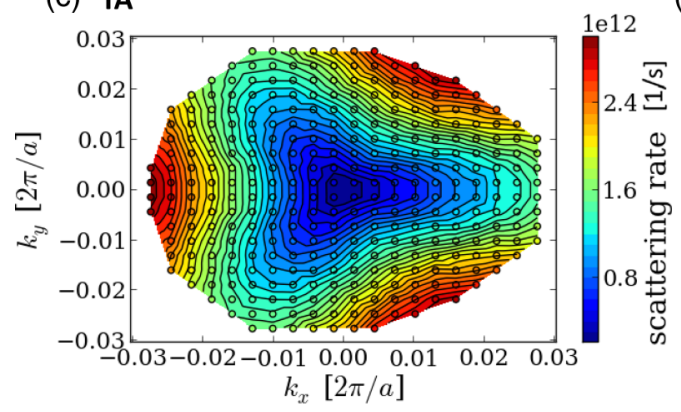

(b)

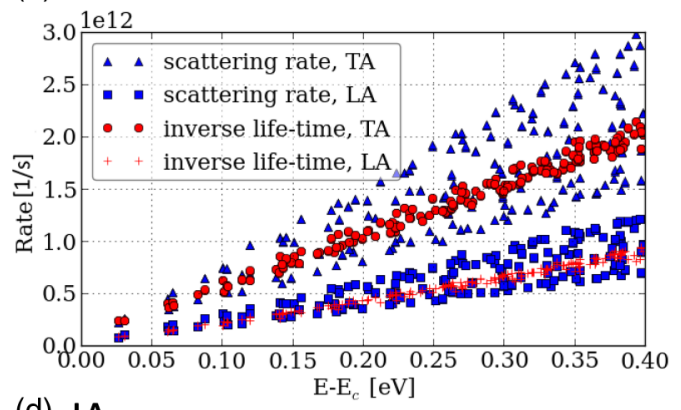

(d) LA

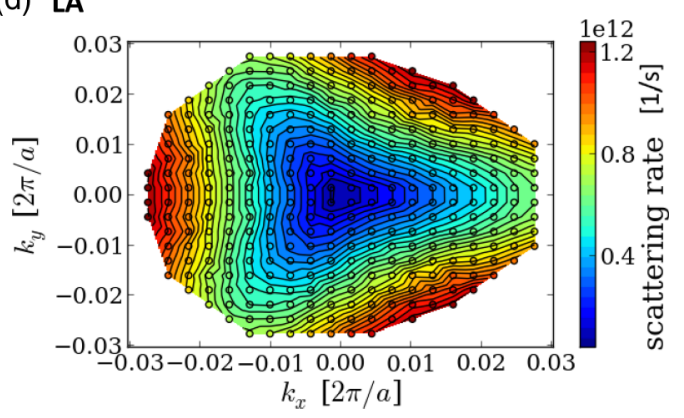

FIG. 7. Scattering rate as a function of energy/k for graphene. (a) At a temperature of $5.7 \mathrm{~K}$ and a Fermi level $\mu_{F}=100 \mathrm{meV}$ along the $\mathbf{k}$ points from $K$ to $\Gamma$ up to $0.5 \mathrm{eV}$. (b) Comparison of the anisotropy in the scattering rate and inverse lifetime at a temperature of $300 \mathrm{~K}$ and a Fermi level $\mu_{F}=100 \mathrm{meV}$. (c), (d) Two-dimensional scattering rate for the highly anisotropic TA and LA phonon modes. The $\mathbf{k}$ points are relative to the Dirac point $K$ and the temperature is $300 \mathrm{~K}$ and Fermi level $\mu_{F}=100 \mathrm{meV}$. The points illustrate the original mesh up to $0.5 \mathrm{eV}$ and the contours are obtained from cubic spline interpolation.

(a)
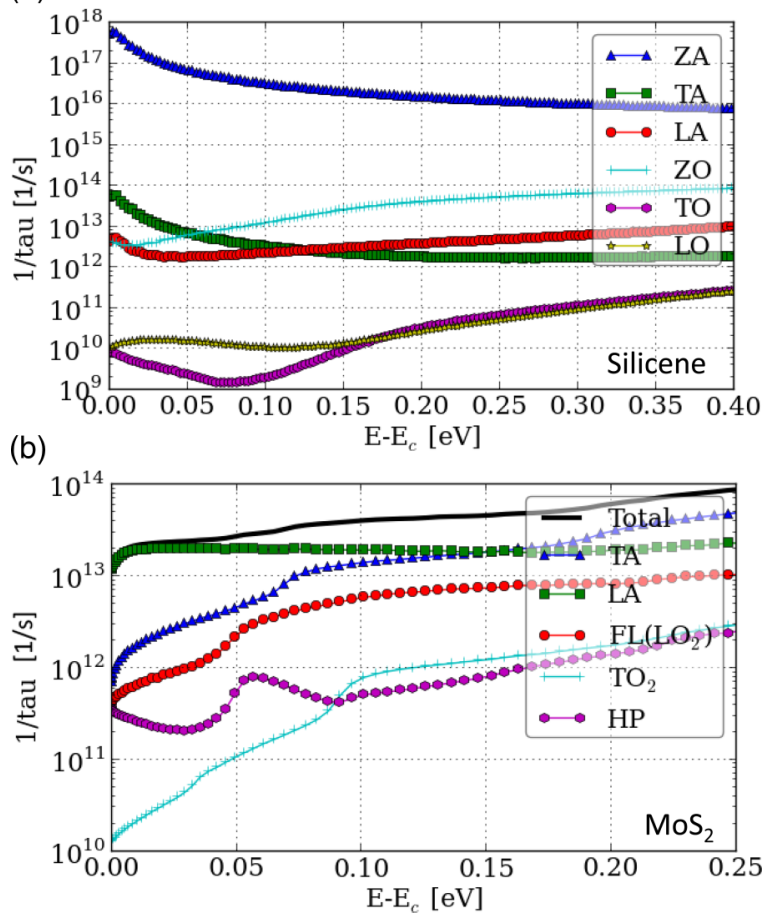

FIG. 8. Scattering rate as a function of energy/k for silicene (a) and $\mathrm{MoS}_{2}$ (b) relative to the conduction band minimum $E_{c}$. In both cases, the temperature is $300 \mathrm{~K}$. The Fermi level is tuned to $\mu_{F}=100 \mathrm{meV}$ for silicene and $500 \mathrm{meV}$ below the conduction band minimum for $\mathrm{MoS}_{2}$. We used $\mathbf{k}$ points along a line from $K$ to $\Gamma$ up to $0.5 \mathrm{eV}$ for silicene and $K$ to $M$ for $\mathrm{MoS}_{2}$. to microscopic changes in potential (deformation potential or nonpolar optical scattering), the scattering related to the macroscopic electric field (piezoelectric or Fröhlich interaction) will be partially screened by a dielectric environment. One approach to handle this difference in screening is to partition electron-phonon interaction in real space into the short-ranged deformation potential contributions and longranged piezoelectric and Fröhlich interactions and apply the screening individually [54]. However, as shown in Fig. 8(b), the Fröhlich interaction $\left(\mathrm{LO}_{2}\right.$ mode) is not dominating the transport and the piezoelectric coupling is most important at low temperatures $[30,60]$.

\section{Mobility}

In Fig. 9, we illustrate the obtained carrier concentration and mobility [79] of graphene, silicene [Figs. 9(a) and 9(b)], and $\mathrm{MoS}_{2}$ [Figs. 9(c) and 9(d)]. We only include intravalley scattering in the present analysis. The same $\mathbf{q}$ mesh is used as for the scattering rate analysis. For the $\mathbf{k}$ space we evaluate the scattering rate in the $\mathbf{k}$ points that contribute, from a very dense $1500 \times 1500$ Monkhorst-Pack sampling of the first Brillouin zone, up to a given Fermi level/carrier density. As an example, the resulting $\mathbf{k}$ points are illustrated by markers in Fig. 7 in the case of graphene. The resulting number of $\mathbf{k}$ points treated within the valley is 316,378 , and 514 for graphene, silicene, and $\mathrm{MoS}_{2}$, respectively.

The phonon-scattering-limited mobilities calculated here show that graphene can have a mobility close to $10^{6} \mathrm{~cm}^{2} / \mathrm{V} \mathrm{s}$ at $100 \mathrm{~K}$ and a carrier density of $3 \times 10^{11} \mathrm{~cm}^{-2}$ (cf. Fig. 9). At $300 \mathrm{~K}$, we obtain a mobility decreasing from $145000 \mathrm{~cm}^{2} / \mathrm{V} \mathrm{s}$ at a carrier density of $1 \times 10^{12} \mathrm{~cm}^{-2}$ to $55000 \mathrm{~cm}^{2} / \mathrm{V} \mathrm{s}$ at a 
(a)

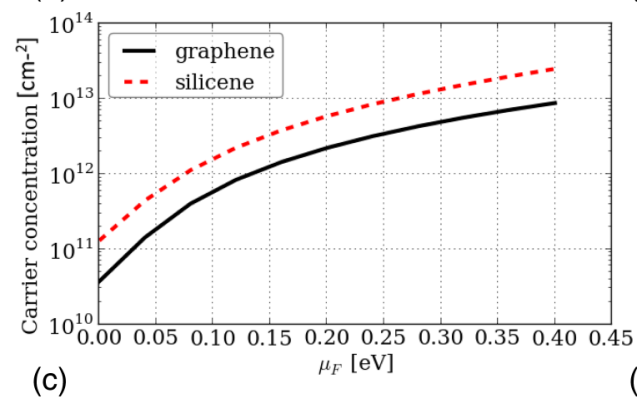

(c)

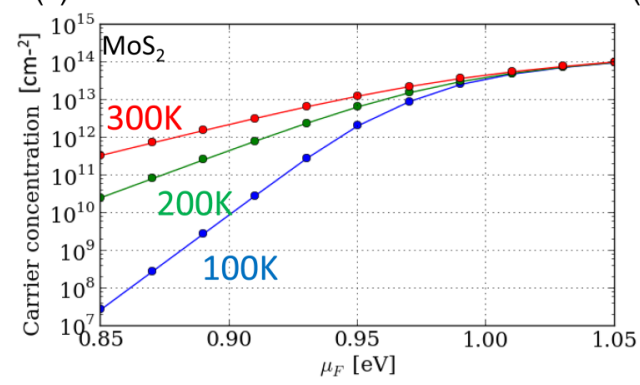

(b)

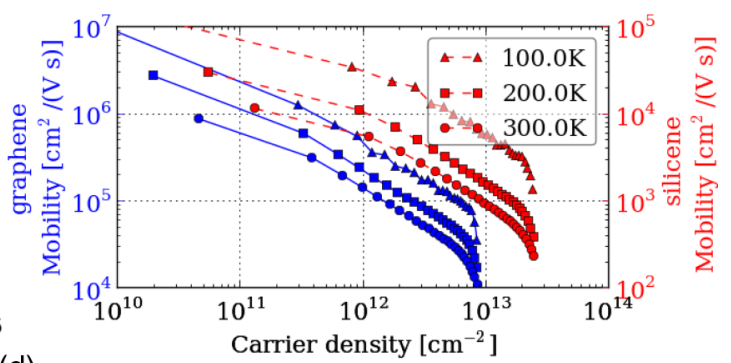

d)

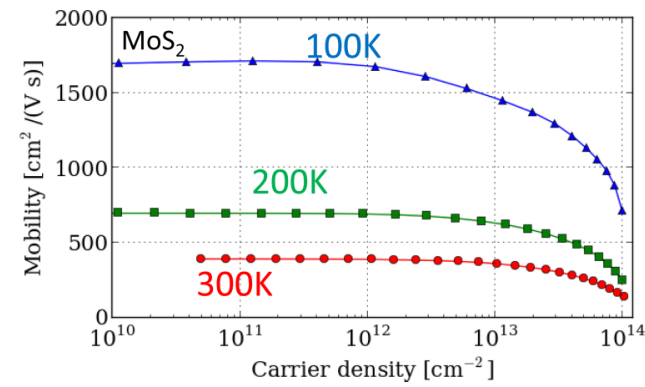

FIG. 9. Carrier concentration as a function of Fermi level for graphene and silicene (a) and $\mathrm{MoS}_{2}$ (c). The conduction band edge of $\mathrm{MoS}_{2}$ is at $0.95 \mathrm{eV}$. The mobility as a function of carrier density is also shown for graphene (b) and silicene (b, inset) and $\mathrm{MoS}_{2}(\mathrm{~d})$.

carrier density of $3 \times 10^{12} \mathrm{~cm}^{-2}$. In comparison, experiments have so far achieved room-temperature values decreasing from roughly $90000 \mathrm{~cm}^{2} / \mathrm{V}$ s to $45000 \mathrm{~cm}^{2} / \mathrm{V} \mathrm{s}$ at the same carrier densities [23]. The same experiment also obtains mobilities up to $10^{6} \mathrm{~cm}^{2} / \mathrm{V} \mathrm{s}$ at lower temperatures. In addition, we find the mobility of silicene to be more than an order of magnitude lower than graphene but still very high if the ZA coupling can be reduced to zero by clamping or other means. We obtain a mobility of roughly $2100 \mathrm{~cm}^{2} / \mathrm{V} \mathrm{s}$ at $300 \mathrm{~K}$ and a carrier density of $3 \times 10^{12} \mathrm{~cm}^{-2}$. Previous calculations have only considered ungated silicene while neglecting the ZA coupling and obtained values in the range of $10-1000 \mathrm{~cm}^{2} / \mathrm{V}$ s at $300 \mathrm{~K}$ $[47,80]$. However, if the ZA coupling cannot be reduced, we find that the mobility will be two to three orders of magnitude lower depending on the carrier density. Specifically, we obtain values of $10 \mathrm{~cm}^{2} / \mathrm{V} \mathrm{s}\left(4 \mathrm{~cm}^{2} / \mathrm{V} \mathrm{s}\right)$ at a temperature of $300 \mathrm{~K}$ $(100 \mathrm{~K})$ and a carrier density of $3 \times 10^{12} \mathrm{~cm}^{-2}$. These findings strongly support the general conclusions made in Ref. [78]. The only experiment on silicene presently published achieves a mobility of roughly $100 \mathrm{~cm}^{2} / \mathrm{V} \mathrm{s}$ at $300 \mathrm{~K}$ [4]. For $\mathrm{MoS}_{2}$, our calculated mobility decreases from a value of $1700 \mathrm{~cm}^{2} / \mathrm{V} \mathrm{s}$ at $100 \mathrm{~K}$ to approximately $400 \mathrm{~cm}^{2} / \mathrm{V} \mathrm{s}$ at $300 \mathrm{~K}$. These results are in good agreement with published first-principles simulations and experimental values [51,60,62]. In comparison, experiments have achieved up to $200 \mathrm{~cm}^{2} / \mathrm{V} \mathrm{s}$ at $300 \mathrm{~K}$ [25].

We note that the electron-phonon coupling as a function of $\mathbf{q}$, as was shown for all materials for a single $\mathbf{k}$ point, was evaluated at all $\mathbf{k}$ points in the mobility calculations in Fig. 9. Another approach that is often applied is to evaluate the scattering rate along a single $\mathbf{k}$ line obtained from a $\left|g_{\mathbf{k q}}^{\lambda n n^{\prime}}\right|$ at a fixed $\mathbf{k}$ point. This can be transformed to a generic energy dependence which is used to evaluate the mobility. This approach, however, neglects part of the anisotropy in the bulk electron-phonon coupling, which was included in the present results.

\section{E. Intervalley scattering}

We only included intravalley scattering in the previous figures. However, it is straightforward to evaluate the intervalley scattering rate separately by doing a calculation with the same $\mathbf{k}$ mesh and shifting the $\mathbf{q}$ mesh to $\mathbf{q}+\mathbf{K}$. We have done so for all three materials along a single $\mathbf{k}$ line to evaluate at what temperatures and carrier densities the intervalley scattering might start contributing.

In Fig. 10, we compare the obtained intravalley and intervalley scattering rates summed over all modes, except the intravalley ZA mode as discussed previously. For graphene, we find that intervalley scattering starts contributing around 200-300 K and at a Fermi level above $140 \mathrm{meV}$, corresponding to a carrier density of $n_{0} \approx 10^{12} \mathrm{~cm}^{-2}$ [cf. Fig. 9(a)]. The reason is that intervalley scattering requires a phonon momentum connecting the two valleys $|\mathbf{q}| \approx\left|\mathbf{K}-\mathbf{K}^{\prime}\right| \approx|\mathbf{K}|$ where the lowest-energy phonon modes ( $\mathrm{ZA}$ and $\mathrm{ZO}$ around

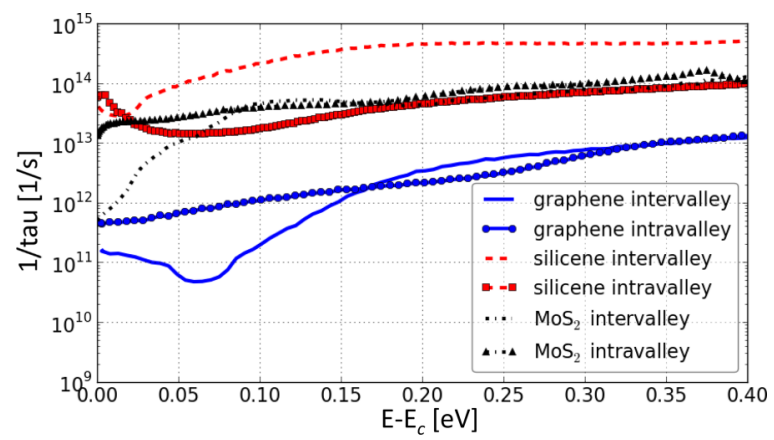

FIG. 10. Scattering rate as a function of energy/k for graphene, silicene, and $\mathrm{MoS}_{2}$ relative to the conduction band minimum $E_{c}$. In all cases, the temperature is $300 \mathrm{~K}$. The Fermi level and $\mathbf{k}$ route are the same as for the intravalley scattering rates presented in Figs. 7(b) and 8 . 
$\mathbf{q}=\mathbf{K}$ ) only exist above $65 \mathrm{meV}$ in the case of graphene [cf. Fig. 3(b)]. A similar onset exists for silicene and $\mathrm{MoS}_{2}$ where we at $300 \mathrm{~K}$ find from Fig. 10 that intervalley scattering starts contributing at a Fermi level of 30 and $100 \mathrm{meV}$ above the conduction band minimum, respectively. For $\mathrm{MoS}_{2}$, this corresponds to $n_{0} \approx 10^{14} \mathrm{~cm}^{-2}$ so that intervalley scattering does not contribute significantly, whereas for silicene intervalley scattering seems to make a significant contribution above $n_{0} \approx 0.5 \times 10^{12} \mathrm{~cm}^{-2}$.

Our definition of the onset for intervalley contribution is that we obtain an intervalley scattering rate of the same order of magnitude as for the intravalley scattering. Using Matthiessen's rule $1 / \mu=1 / \mu^{\text {intra }}+1 / \mu^{\text {inter }}$ we can estimate that the total mobility will decrease by approximately a factor of 2 at carrier densities above $n_{0} \approx 10^{12} \mathrm{~cm}^{-2}$ if intervalley scattering was included for graphene. For $\mathrm{MoS}_{2}$, the contribution will be much smaller at assessable carrier densities, whereas silicene in the limit without intravalley ZA coupling can have as much as an order of magnitude lower mobility due to intervalley scattering for carrier densities above $n_{0} \approx 0.5 \times 10^{12} \mathrm{~cm}^{-2}$.

\section{CONCLUSION}

In summary, we have presented a Boltzmann transport equation (BTE) solver implemented in the Atomistix ToolKit (ATK) simulation tool. The method allows for calculation of material properties, including the electron-phonon interaction, from first principles. We have applied the tool to calculate the phonon-limited mobility in $n$-type monolayer graphene, silicene, and $\mathrm{MoS}_{2}$. Our results compare well to published theoretical results and experiments for $\mathrm{MoS}_{2}$ and graphene and extend previous theoretical calculations for silicene, where a significantly lower mobility than previously reported is obtained due to a strong coupling with the flexural (ZA) mode, that was so far neglected. The bulk electron-phonon coupling is highly anisotropic due to scattering suppression in different $q$ directions, as well as a nontrivial $q$ dependence, related to the combined symmetry of the electron and phonon states. The simulations provide an upper bound for the electron mobilities of the selected 2D materials. The ab initio approach demonstrated in this paper can be directly applied to other materials in 1D, 2D, and 3D larger nanostructures and may be straightforwardly extended to study cases with electronimpurity scattering. In addition, we have illustrated how the reduction of the lattice symmetry, when going from graphene to silicene with no planar symmetry and further on to $\mathrm{MoS}_{2}$ with no inversion symmetry, leads to additional scattering mechanisms and modes interacting with the charge carriers. This makes a strong case for the need of a fully numerical solution of the BTE together with a first-principles method to evaluate the bulk electron-phonon interaction, especially, when going to systems with many modes, such as systems with low lattice symmetry, slab structures, and nanostructured materials.

\section{ACKNOWLEDGMENTS}

We thank K. Kaasbjerg (DTU) and T. Frederiksen (San Sebastian) for useful discussions on the methods employed.
The authors acknowledge support from Innovation Fund Denmark, grant Nano-Scale Design Tools for the Semiconductor Industry (Grant No. 79-2013-1). The Center for Nanostructured Graphene (CNG) is sponsored by the Danish National Research Foundation, Project No. DNRF58.

\section{APPENDIX: FINITE-DIFFERENCE DERIVATIVE OF HAMILTONIAN}

The derivative of the Hamiltonian operator is calculated in a way similar to the dynamical matrix following the approach of Kaasbjerg et al. [60]. We construct a supercell by repeating the primitive configuration a number of times in each periodic direction. Subsequently, we displace the atoms in the central cell by $\pm \delta$ in all Cartesian directions (forward and backward) and calculate the derivative with respect to, e.g., atom $i$ in the $x$ direction as

$$
\frac{\partial \hat{H}}{\partial x_{i}} \approx \frac{\hat{H}\left(x_{i}+\delta\right)-\hat{H}\left(x_{i}-\delta\right)}{2 \delta},
$$

where $\hat{H}\left(x_{i}+\delta\right)$ indicates the Hamiltonian operator obtained for the configuration where atom $i$ is displaced by $\delta$ in the positive $x$ direction. Note that Eq. (A1) applies for the Hamiltonian operator, and not for the Hamiltonian matrix expressed in a basis of LCAO basis functions. In the latter case it is necessary to correct also for the displacement of the basis orbitals [68].

The Hamiltonian operator has several terms:

$$
\hat{H}=\hat{T}+\hat{V}_{\text {local }}+\hat{V}_{\mathrm{NL}},
$$

where $\hat{T}$ is the kinetic energy operator, $\hat{V}_{\text {local }}$ is the local potential including the exchange-correlation potential, the Hartree potential, as well as the local pseudopotential, while $\hat{V}_{\mathrm{NL}}$ is the nonlocal Kleinman-Bylander pseudopotential. Since the kinetic energy does not depend on the atomic coordinates, the derivative of that is zero. The derivative of the local potential can be directly evaluated using Eq. (A1). The nonlocal potential is written as

$$
\hat{V}_{\mathrm{NL}}=\sum_{i} \sum_{\alpha \beta}\left|\chi_{\alpha}^{i}\right\rangle v_{\alpha \beta}^{i}\left\langle\chi_{\beta}^{i}\right|,
$$

where $\left|\chi_{\alpha}^{i}\right\rangle$ is a projector function centered on atom $i$ and $v_{\alpha \beta}^{i}$ are (fixed) projector coupling elements. The derivative is written as

$$
\begin{aligned}
\frac{\partial \hat{V}_{\mathrm{NL}}}{\partial x_{j}} & =\frac{\partial}{\partial x_{j}} \sum_{i} \sum_{\alpha \beta}\left|\chi_{\alpha}^{i}\right\rangle v_{\alpha \beta}\left\langle\chi_{\beta}^{i}\right| \\
& =\sum_{\alpha \beta}\left(\frac{\partial\left|\chi_{\alpha}^{j}\right\rangle}{\partial x_{j}} v_{\alpha \beta}\left\langle\chi_{\beta}^{j}|+| \chi_{\alpha}^{j}\right\rangle v_{\alpha \beta} \frac{\partial\left\langle\chi_{\beta}^{j}\right|}{\partial x_{j}}\right) .
\end{aligned}
$$

The derivatives of the projector functions are evaluated numerically as in Eq. (A1).

Having calculated the derivative of the Hamiltonian operator we evaluate the derivative in the LCAO basis

$$
\left(\frac{\partial \mathbf{H}}{\partial x_{i}}\right)_{\mu \nu}=\left\langle\phi_{\mu}\left|\frac{\partial \hat{H}}{\partial x_{i}}\right| \phi_{\nu}\right\rangle .
$$


[1] D. Akinwande, N. Petrone, and J. Hone, Nat. Commun. 5, 5678 (2014).

[2] A. K. Geim and I. V. Grigorieva, Nature (London) 499, 419 (2013).

[3] D. K. Efetov and P. Kim, Phys. Rev. Lett. 105, 256805 (2010).

[4] L. Tao, E. Cinquanta, D. Chiappe, C. Grazianetti, M. Fanciulli, M. Dubey, A. Molle, and D. Akinwande, Nat. Nanotechnol. 10, 227 (2015).

[5] M. Xu, T. Liang, M. Shi, and H. Chen, Chem. Rev. 113, 3766 (2013).

[6] S. Z. Butler, S. M. Hollen, L. Cao, Y. Cui, J. A. Gupta, H. R. Gutiérrez, T. F. Heinz, S. S. Hong, J. Huang, A. F. Ismach, E. Johnston-Halperin, M. Kuno, V. V. Plashnitsa, R. D. Robinson, R. S. Ruoff, S. Salahuddin, J. Shan, L. Shi, M. G. Spencer, M. Terrones, W. Windl, and J. E. Goldberger, ACS Nano 7, 2898 (2013).

[7] B. Feng, Z. Ding, S. Meng, Y. Yao, X. He, P. Cheng, L. Chen, and K. Wu, Nano Lett. 12, 3507 (2012).

[8] A. Kara, H. Enriquez, A. P. Seitsonen, L. C. Lew Yan Voon, S. Vizzini, B. Aufray, and H. Oughaddou, Surf. Sci. Rep. 67, 1 (2012).

[9] M. Houssa, A. Dimoulas, and A. Molle, J. Phys.: Condens. Matter 27, 253002 (2015).

[10] S. Balendhran, S. Walia, H. Nili, S. Sriram, and M. Bhaskaran, Small 11, 640 (2015).

[11] J. L. Manñes, Phys. Rev. B 76, 045430 (2007).

[12] T. Stauber, N. M. R. Peres, and F. Guinea, Phys. Rev. B 76, 205423 (2007).

[13] E. H. Hwang and S. Das Sarma, Phys. Rev. B 77, 115449 (2008).

[14] R. S. Shishir and D. K. Ferry, J. Phys.: Condens. Matter 21, 344201 (2009).

[15] R. S. Shishir and D. K. Ferry, J. Phys.: Condens. Matter 21, 232204 (2009).

[16] J. H. Chen, C. Jang, M. Ishigami, S. Xiao, W. G. Cullen, E. D. Williams, and M. S. Fuhrer, Solid State Commun.149, 1080 (2009).

[17] K. Kaasbjerg, K. S. Thygesen, and K. W. Jacobsen, Phys. Rev. B 85, 165440 (2012).

[18] M. Lazzeri and F. Mauri, Phys. Rev. Lett. 97, 266407 (2006).

[19] C.-H. Park, F. Giustino, M. L. Cohen, and S. G. Louie, Phys. Rev. Lett. 99, 086804 (2007)

[20] S. Pisana, M. Lazzeri, C. Casiraghi, K. S. Novoselov, A. K. Geim, A. C. Ferrari, and F. Mauri, Nat. Mater. 6, 198 (2007).

[21] M. Calandra and F. Mauri, Phys. Rev. B 76, 205411 (2007).

[22] C.-H. Park, F. Giustino, C. D. Spataru, M. L. Cohen, and S. G. Louie, Phys. Rev. Lett. 102, 076803 (2009).

[23] L. Wang, I. Meric, P. Y. Huang, Q. Gao, Y. Gao, H. Tran, T. Taniguchi, K. Watanabe, L. M. Campos, D. A. Muller, J. Guo, P. Kim, J. Hone, K. L. Shepard, and C. R. Dean, Science 342, 614 (2013).

[24] X. Cui, G.-H. Lee, Y. D. Kim, G. Arefe, P. Y. Huang, C.-H. Lee, D. A. Chenet, X. Zhang, L. Wang, F. Ye, F. Pizzocchero, B. S. Jessen, K. Watanabe, T. Taniguchi, D. A. Muller, T. Low, P. Kim, and J. Hone, Nat. Nanotechnol. 10, 534 (2015).

[25] B. Radisavljevic, A. Radenovic, J. Brivio, V. Giacometti, and A. Kis, Nat. Nanotechnol. 6, 147 (2011).

[26] A. K. M. Newaz, Y. S. Puzyrev, B. Wang, S. T. Pantelides, and K. I. Bolotin, Nat. Commun. 3, 734 (2012).
[27] F. Chen, J. Xia, and N. Tao, Nano Lett. 9, 1621 (2009).

[28] T. Ando, A. B. Fowler, and F. Stern, Rev. Mod. Phys. 54, 437 (1982).

[29] S. Das Sarma, S. Adam, E. H. Hwang, and E. Rossi, Rev. Mod. Phys. 83, 407 (2011).

[30] T. Kawamura and S. Das Sarma, Phys. Rev. B 45, 3612 (1992).

[31] M. V. Fischetti and S. E. Laux, J. Appl. Phys. 80, 2234 (1996).

[32] T. Fang, A. Konar, H. Xing, and D. Jena, Phys. Rev. B 84, 125450 (2011).

[33] M. V. Fischetti and S. E. Laux, Phys. Rev. B 48, 2244 (1993).

[34] E. H. Hwang and S. Das Sarma, Phys. Rev. B 79, 165404 (2009).

[35] M. A. Kuroda, J. Tersoff, and G. J. Martyna, Phys. Rev. Lett. 106, 116804 (2011).

[36] E. Mariani and F. von Oppen, Phys. Rev. Lett. 100, 076801 (2008).

[37] N. Sule and I. Knezevic, J. Appl. Phys. 112, 053702 (2012).

[38] Y.-M. Niquet, C. Delerue, D. Rideau, and B. Videau, IEEE Trans. Electron Devices 59, 1480 (2012).

[39] S. Cauley, M. Luisier, V. Balakrishnan, G. Klimeck, and C.-K. Koh, J. Appl. Phys. 110, 043713 (2011).

[40] V. Perebeinos, J. Tersoff, and P. Avouris, Phys. Rev. Lett. 109, 236604 (2012).

[41] T. Gunst, J.-T. Lü, P. Hedegård, and M. Brandbyge, Phys. Rev. B 88, 161401 (2013).

[42] J.-T. Lü, M. Brandbyge, P. Hedegård, T. N. Todorov, and D. Dundas, Phys. Rev. B 85, 245444 (2012).

[43] C.-H. Park, N. Bonini, T. Sohier, G. Samsonidze, B. Kozinsky, M. Calandra, F. Mauri, and N. Marzari, Nano Lett. 14, 1113 (2014).

[44] T. Sohier, M. Calandra, C.-H. Park, N. Bonini, N. Marzari, and F. Mauri, Phys. Rev. B 90, 125414 (2014).

[45] Z. Wang, S. Wang, S. Obukhov, N. Vast, J. Sjakste, V. Tyuterev, and N. Mingo, Phys. Rev. B 83, 205208 (2011).

[46] O. D. Restrepo, K. Varga, and S. T. Pantelides, Appl. Phys. Lett. 94, 212103 (2009)

[47] X. Li, J. T. Mullen, Z. Jin, K. M. Borysenko, M. Buongiorno Nardelli, and K. W. Kim, Phys. Rev. B 87, 115418 (2013).

[48] Z.-G. Shao, X.-S. Ye, L. Yang, and C.-L. Wang, J. Appl. Phys. 114, 093712 (2013).

[49] A. Szabo, R. Rhyner, and M. Luisier, Phys. Rev. B 92, 035435 (2015).

[50] K. M. Borysenko, J. T. Mullen, E. A. Barry, S. Paul, Y. G. Semenov, J. M. Zavada, M. B. Nardelli, and K. W. Kim, Phys. Rev. B 81, 121412 (2010).

[51] O. D. Restrepo, K. E. Krymowski, J. Goldberger, and W. Windl, New J. Phys. 16, 105009 (2014).

[52] J.-A. Yan, R. Stein, D. M. Schaefer, X.-Q. Wang, and M. Y. Chou, Phys. Rev. B 88, 121403 (2013).

[53] P. D. Yoder, V. D. Natoli, and R. M. Martin, J. Appl. Phys. 73, 4378 (1993).

[54] K. Kaasbjerg, K. S. Thygesen, and A.-P. Jauho, Phys. Rev. B 87, 235312 (2013).

[55] M. Lazzeri, S. Piscanec, F. Mauri, A. C. Ferrari, and J. Robertson, Phys. Rev. B 73, 155426 (2006).

[56] F. Mazzola, J. W. Wells, R. Yakimova, S. Ulstrup, J. A. Miwa, R. Balog, M. Bianchi, M. Leandersson, J. Adell, P. Hofmann, and T. Balasubramanian, Phys. Rev. Lett. 111, 216806 (2013). 
[57] J. D. Buron, F. Pizzocchero, P. U. Jepsen, D. H. Petersen, J. M. Caridad, B. S. Jessen, T. J. Booth, and P. Bøggild, Sci. Rep. 5, 12305 (2015).

[58] F. Giustino, M. L. Cohen, and S. G. Louie, Phys. Rev. B 76, 165108 (2007).

[59] M. V. Fischetti, J. Kim, S. Narayanan, Z.-Y. Ong, C. Sachs, D. K. Ferry, and S. J. Aboud, J. Phys.: Condens. Matter 25, 473202 (2013).

[60] K. Kaasbjerg, K. S. Thygesen, and K. W. Jacobsen, Phys. Rev. B 85, 115317 (2012).

[61] S. Piscanec, M. Lazzeri, F. Mauri, A. C. Ferrari, and J. Robertson, Phys. Rev. Lett. 93, 185503 (2004).

[62] W. Li, Phys. Rev. B 92, 075405 (2015).

[63] Atomistix ToolKit, version 2015, QuantumWise A/S.

[64] G. D. Mahen, Many-Particle Physics, 3rd ed. (Plenum, New York, 2000).

[65] Z. Stanojevic, O. Baumgartner, M. Karner, L. Filipovic, C. Kernstock, and H. Kosina, in 2014 International Conference on Simulation of Semiconductor Processes and Devices (SISPAD) (IEEE, Piscataway, NJ, 2014), pp. 181-184.

[66] The scattering angle is derived as follows. We define the angles $\hat{\mathbf{v}}_{\mathbf{k} n} \cdot \hat{\mathbf{v}}_{\mathbf{k}^{\prime} n^{\prime}} \equiv \cos (\theta)$ and $n_{\mathbf{k} n}=\hat{\mathbf{E}} \cdot \hat{\mathbf{v}}_{\mathbf{k} n} \equiv \cos \left(\theta^{\prime}\right)$. Applying the spherical law of cosines [64] we can write $n_{\mathbf{k}^{\prime} n^{\prime}}=\hat{\mathbf{E}} \cdot \hat{\mathbf{v}}_{\mathbf{k}^{\prime} n^{\prime}}=$ $\cos (\theta) \cos \left(\theta^{\prime}\right)+\sin (\theta) \sin \left(\theta^{\prime}\right) \cos (\phi)$. The term with $\cos (\phi)$ will vanish when doing the $\mathbf{k}^{\prime}$ integration since no other parameter depends on $\phi$. Therefore, we obtain $\cos (\theta)=\frac{n_{\mathbf{k}^{\prime} \mathbf{n}^{\prime}}}{\mathrm{n}_{\mathbf{k}}}$.

[67] E. H. Hwang and S. Das Sarma, Phys. Rev. B 77, 195412 (2008).

[68] T. Frederiksen, M. Paulsson, M. Brandbyge, and A.-P. Jauho, Phys. Rev. B 75, 205413 (2007).
[69] The sign in the phase factors should be consistent with the directions applied in the phonon and electronic-structure calculations of the code under consideration. We note that the momentum conservation rule $\delta_{\mathbf{k}^{\prime}, \mathbf{k}+\mathbf{q}}$ comes out of the momentum matrix element $g_{\mathbf{k} \mathbf{k}^{\prime} \mathbf{q}}^{\lambda n^{\prime}}$. Correspondingly, the opposite selection rule $\delta_{\mathbf{k}^{\prime}, \mathbf{k}-\mathbf{q}}$ is obtained from the matrix element $g_{\mathbf{k}^{\prime} \mathbf{k} \mathbf{q}}^{\lambda \lambda n^{\prime}}$. Since $\left|g_{\mathbf{k q}}^{\lambda n n^{\prime}}\right|=\left|g_{\mathbf{q k}}^{\lambda n n^{\prime}}\right|$ only one element needs to be evaluated in the calculation of the mobility.

[70] X. Gu and R. Yang, J. Appl. Phys. 117, 025102 (2015).

[71] K. F. Mak, C. Lee, J. Hone, J. Shan, and T. F. Heinz, Phys. Rev. Lett. 105, 136805 (2010).

[72] A. Splendiani, L. Sun, Y. Zhang, T. Li, J. Kim, C.-Y. Chim, G. Galli, and F. Wang, Nano Lett. 10, 1271 (2010).

[73] R. Fivaz and E. Mooser, Phys. Rev. 163, 743 (1967).

[74] X. Zhang, X.-F. Qiao, W. Shi, J.-B. Wu, D.-S. Jiang, and P.-H. Tan, Chem. Soc. Rev. 44, 2757 (2015).

[75] A. Molina-Saánchez and L. Wirtz, Phys. Rev. B 84, 155413 (2011).

[76] J. Sjakste, N. Vast, M. Calandra, and F. Mauri, Phys. Rev. B 92, 054307 (2015).

[77] C. Verdi and F. Giustino, Phys. Rev. Lett. 115, 176401 (2015).

[78] M. V. Fischetti and W. G. Vandenberghe, arXiv:1511.06796v1.

[79] The mobility calculations were carried out on a cluster of 128 CPUs (Intel Xeon E5-series) and took approximately 24 hours for each material. Most of the time was used for evaluating the bulk electron-phonon interaction in the $\mathbf{k}$ and $\mathbf{q}$ space, which however shows close to linear speedup.

[80] R. Wang, X. Pi, Z. Ni, Y. Liu, S. Lin, M. Xu, and D. Yang, Sci. Rep. 3, 3507 (2013). 